\title{
Taking culture seriously in biomedical HIV prevention trials: A meta-synthesis of qualitative studies
}

Clara Rubincam, Ashley Lacombe-Duncan \& Peter A. Newman

Version Post-Print/ Accepted Manuscript

Citation Rubincam, C., Lacombe-Duncan, A. and Newman, P.A. (2016). Taking (published version) culture seriously in biomedical HIV prevention trials: a meta-synthesis of qualitative studies. Expert Review of V accines 15(3): 331-347. doi: 10.1586/14760584.2016.1118349

Publisher's Statement This is an Accepted Manuscript of an article published by Taylor \& Francis in the American Journal of Bioethics in 2016, available at http://www.tandfonline.com/doi/full/10.1586/14760584.2016.1118349 
NOTE: This document is the authors' post-print (post-refereeing) version. The final publication is available at: http://www.tandfonline.com/doi/full/10.1586/14760584.2016.1118349

\section{Citation:}

Rubincam, C., Lacombe-Duncan, A., \& Newman, P. A. (2015). Taking culture seriously in biomedical HIV prevention trials: a meta-synthesis of qualitative studies. Expert Review of Vaccines, 15(3), 331-347. DOI: $\underline{10.1586 / 14760584.2016 .1118349}$

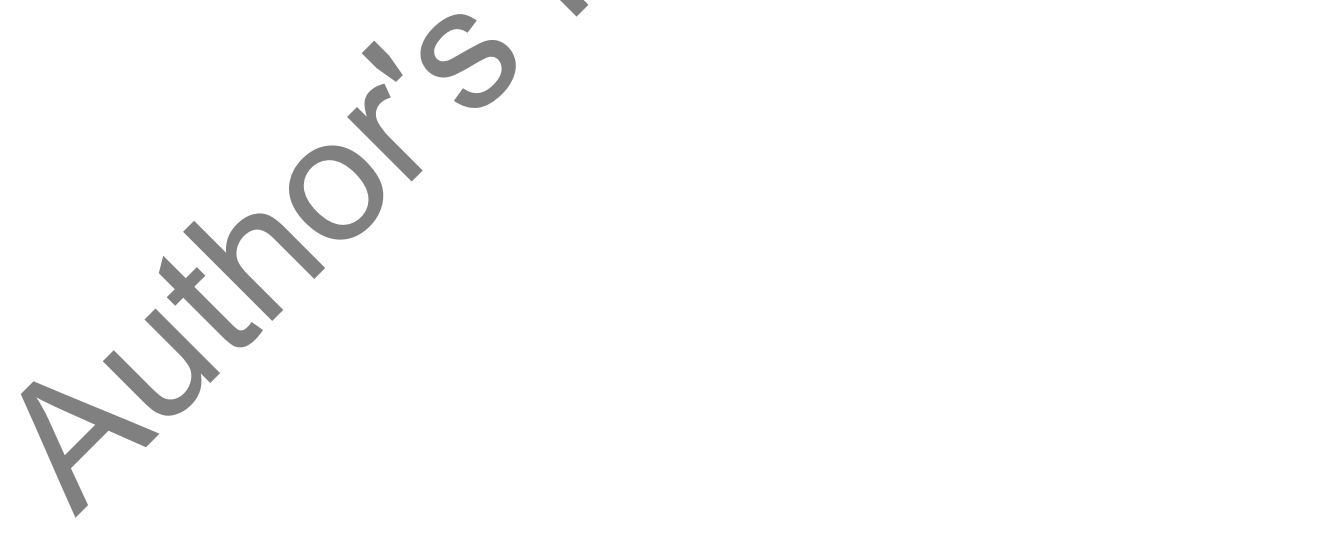




\section{Taking culture seriously in biomedical HIV prevention trials: a meta-synthesis of qualitative studies}

Clara Rubincam, Ashley Lacombe-Duncan and Peter A. Newman Factor-Inwentash Faculty of Social Work, University of Toronto, Toronto, Canada

\section{Abstract}

A substantial gap exists between widespread acknowledgement of the importance of incorporating cultural sensitivity in biomedical HIV prevention trials and empirical evidence to guide the operationalization of cultural sensitivity in these trials. We conducted a systematic literature search and qualitative meta-synthesis to explore how culture is conceptualized and operationalized in global biomedical HIV prevention trials. Across 29 studies, the majority $(\mathrm{n}=$ 17) were conducted in resource-limited settings. We identified four overarching themes: (1) semantic cultural sensitivity - challenges in communicating scientific terminology into local vernaculars; (2) instrumental cultural sensitivity - understanding historical experiences to guide tailoring of trial activities; (3) budgetary, logistical, and personnel implications of operationalizing cultural sensitivity; and (4) culture as an asset. Future investigations should address how sociocultural considerations are operationalized across the spectrum of trial preparedness, implementation, and dissemination in particular sociocultural contexts, including intervention studies and evaluations of the effectiveness of methods used to operationalize culturally sensitive practices. 


\section{Introduction}

The importance of addressing cultural contexts in biomedical HIV prevention trials has been advanced in several studies [1-5] and policy documents [6-9]. In recognition of concerns about the ethical conduct of biomedical HIV prevention trials in lowand middleincome countries, researchers have been encouraged to adopt UNAIDS/AVAC's Good Participatory Practice (GPP) guidelines [7]. GPP outlines six guiding principles to improve the ethical conduct of clinical trials in affected communities [7]. An adjunct document, the GPP Blueprint for Stakeholder Engagement [10], provides a step-by-step guide for designing a comprehensive engagement plan for community stakeholders. Both of these documents, and others like them, emphasize the importance of tailoring trial activities to the particular sociocultural context of a study site: by ensuring that consent procedures align with cultural norms, that research questions and procedures are culturally sensitive and appropriate, that researchers develop sociocultural competency in the particular research context, that the study remain sensitive to issues that may arise in the course of research that violate certain sociocultural taboos, and that dissemination activities are conducted in a transparent manner in order to build trust and lay a positive foundation for future research' [7, p.61].

A sophisticated literature addresses the adaptation of informed consent processes for diverse cultural contexts, pointing to circumstances that may affect the extent to which participants are meaningfully informed. These include differences in understandings of disease and conditions that may limit the voluntary nature of consent, such as limited access to health services [11-19]. Many of these studies engage in in-depth exploration of particular sociocultural contexts so that information and consent materials can better draw on local meanings, analogies, and images. For instance, Corneli and colleagues [11] describe the steps taken to develop a context-specific informed consent procedure for a clinical trial in Malawi. After several formative research studies undertaken to determine what participants understood about medical research, they developed 
counseling flip charts using analogies for scientific terminology that were deemed meaningful to local populations. Though researchers were informed that in the local language, Chichewa, there is no word for 'experiment', they drew on community members' longstanding expertise with agriculture to discuss several common concepts in medical research [11]. In doing so, researchers rendered unfamiliar concepts in familiar ways, building on existing sources of knowledge to improve trust and understanding in the clinical trial process.

Beyond studies focused on informed consent, however, relatively few investigations have demonstrated or assessed how sociocultural sensitivity is operationalized in actual trial activities, such as stakeholder engagement, participant recruitment, data collection, and dissemination of trial results. As a result, there remains a substantial gap between the widespread acknowledgment of the importance of tailoring trial activities to the particular sociocultural specificities of trial sites, and empirical evidence on what is actually required logistically, budgetarily, and conceptually to achieve this objective.

In an effort to synthesize insights from existing research, we conducted a meta-synthesis of qualitative studies of biomedical HIV prevention research with a focus on how cultural considerations were accommodated and addressed during formative and in-process activities for biomedical HIV prevention trials.

\section{Defining the study}

Part of the challenge in defining which studies to include in our assessment of sociocultural and contextual sensitivity is that, as others have acknowledged in research on informed consent, 'the meaning of "cultural sensitivity" is itself highly contested' [20]. This has implications for the implementation of a range of research activities. 
Bayer [21, p.896] helpfully distinguishes between three different conceptualizations of cultural sensitivity: the semantic - underscoring the importance of adapting HIV prevention messages to the particular linguistic and stylistic conventions of a group; the instrumental - highlighting the importance of understanding the unique contexts in which trials take place so as to appropriately tailor trial activities; and the principled - which prohibits 'those interventions that violate the cultural norms of those to whom they are directed' [21, p.896]^Bayer strongly supports the former two while highlighting problems with the latter, such as the potential that principled constructions of cultural sensitivity will be used to elevate the cultural norms of a particular group as beyond reproach - even in cases when such norms lead to harm. He criticizes, for instance, the opposition to condom distribution and condom education for high school students by some parents who were more aligned with religious and/or conservative ideologies, even as principled interpretations might suggest that public health authorities should unilaterally respect the cultural norms of these communities [21].

Accordingly, in the present analysis we draw on the semantic and instrumental conceptualizations of cultural sensitivity to review and synthesize processes and findings from research that seeks either to improve the content or style of messaging around biomedical HIV prevention technologies and trials, or to enhance understanding of the sociocultural context of participants and the participating community. With the goal of advancing the implementation of cultural sensitivity in biomedical HIV prevention trials, we address the following questions: how has culture been conceptualized and operationalized in regard to biomedical HIV prevention trials? What activities are being undertaken to address cultural sensitivity in biomedical HIV prevention trials? 


\section{Methods}

Qualitative meta-synthesis involves conducting a systematic review of qualitative investigations on a particular topic; this is followed by an iterative process of data synthesis whereby individual qualitative studies are integrated, generating new themes from combined qualitative study findings [22]. Individual qualitative studies may include phenomenological, ethnographic, grounded theory, and other interpretive or descriptive research findings [22]. Through a process of meta-synthesis, we aim to advance understanding of how the mandate of cultural sensitivity is being conceptualized and operationalized (or not) in biomedical HIV prevention trials and what activities are undertaken to address cultural sensitivity within these trials.

We apply Sandelowski and Barroso's [22] metasynthesis stages, which include (1) forming a research question, (2) systematically searching the literature, (3) selecting articles for study inclusion, (4) critically appraising the quality of included studies, (5) abstracting data from the studies, and (6) synthesizing the findings.

Meta-synthesis has been applied within the broad clinical trial literature to understand willingness to participate in Clinical trials among patients of Chinese heritage [23] and to various HIV-related research questions: how do the social determinants of health influence AfricanAmericans living with HIV [24]? What are the commonalities and differences in infant feeding attitudes in the context of HIV in sub-Saharan Africa [25]? How do HIV-positive women experience motherhood [26]?

\section{Literature search}

We developed a search strategy that included searching eight electronic databases to reflect a broad spectrum of disciplines related to health research: OVID: Medline, EMBASE; EBSCO: CINAHL, and ProQuest: Applied Social Sciences Index and Abstracts (ASSIA), ERIC, 
PsycINFO, Social Services Abstracts and Sociological Abstracts from inception until June 2015. Key search words were (1) vaccine or pharmaceutical; and (2) trial or develop or clinical trial; and (3) culture or history/historical or context or lesson or mistrust or misunderstanding; and (4) case study or qualitative or mixed methods. The broad search terms were developed after reviewing keywords from studies identified through a preliminary search and from the personal libraries of two authors (PN and $\mathrm{CR}$ ), whose primary research focus is on social and behavioral considerations in the development, testing and implementation of new 1 HV prevention technologies. The broad range of terms was chosen to reflect the diversity in HIV prevention technologies (e.g. vaccines, microbicides, antiretroviral therapy) and the various ways in which researchers discuss and situate culture (e.g. utilizing 'context' and 'culture' interchangeably or focusing on trial mistrust as a cultural consideration).

The search was not limited to qualitative studies to ensure that qualitative data available in mixed methods studies could be identified. Consistent with other studies involving synthesis of qualitative research [27], we opted to exclude gray literature and dissertations. We complemented the systematic search by utilizing snowballing techniques, including reviewing reference lists of included articles and review articles, and electronic citation tracking. These techniques are commonly used in developing a comprehensive list of primary studies for qualitative synthesis [28]. Lastly, we searched Google Scholar for any additional articles. All abstracts and relevant articles were reviewed by two investigators (CR and ALD), with a third (PN) available to resolve discrepancies.

\section{Study selection}

We selected qualitative studies that used in-depth interviews, focus groups or case study methodology if they (1) incorporated discussion of social, contextual or cultural considerations in HIV prevention technology research from the perspective of trial volunteers, key stakeholders 
from communities (e.g. community leaders, potential volunteers) and/or trial staff in locations in which a trial took place or was planned; and (2) were published in English in peer-reviewed journals. We excluded studies that were not HIV prevention-technology specific (e.g. about other pharmaceutical clinical trials), that focused on informed consent or on acceptability of future/hypothetical HIV vaccines, and that were not in the context of planned for, ongoing, or closed trials. We utilized the Preferred Reporting Items for Systematic Review and Metaanalysis flowchart (Figure 1) [29] to track the study selection process.

\section{Quality of reporting}

We used the Consolidated Criteria for Reporting Qualitative Research (COREQ) 32-item checklist to assess the quality of reporting of each included study. The COREQ assesses three key areas: research team and reflexivity, study design, and data analysis and reporting. The COREQ aims to promote transparency in research methods and duplication of qualitative studies [30]. However, as the COREQ is a relatively new measure, and qualitative research is not typically published on the basis of achieving baseline comparable standards, no studies were excluded on the basis of the COREQ assessment. COREQ results are presented to support transparency and rigorin this meta-synthesis.

\section{Data abstraction}

Two reviewers, (CR and ALD) read each included study thoroughly and independently abstracted data from included articles. We abstracted the following study characteristics: author names, country where the study was conducted, number and type of participants (e.g. trial participant, community stakeholder, trial staff), trial status (in preparation, ongoing, closed), data analysis technique(s) used, and primary research question. 


\section{Data synthesis}

Our primary method of data analysis was thematic synthesis,[31] adapted to include elements of Noblit and Hare's [32] meta-ethnography methods, and informed by metadata synthesis techniques [33]. First, two authors (CR and ALD) read each study thoroughly and multiple times to understand the overall study. Second, two authors (CR and ALD) coded the introduction, methods, results, and discussion sections from each study to develop descriptive themes related to the discussion of culture in included studies. Because the focus of this meta-synthesis is on how biomedical HIV prevention technology trials conceptualize and integrate culture throughout study processes, we reviewed how authors frame studies (introduction) and types of methods utilized (methods), in addition to results and discussion sections. Quotations, with identifiers, were extracted from participants and investigators to support descriptive themes. We (CR, ALD, and PN) then compared and contrasted descriptive themes across studies and developed analytic themes, which support new interpretations of the combined original data [31].

\section{Results}

\section{Study characteristics}

Of the 42 articles reviewed in full, 29 articles - representing 13 ongoing trials, 5 closed trials, 9 trials in preparation, and 2 drawing on data from more than one trial at different stages - were included. While not the explicit primary objective of all studies, these studies each reported findings on how sociocultural considerations were addressed in biomedical HIV prevention trials. The majority of studies were conducted in lowand middle-income countries $(n=17)$, with just over one-third $(n=10)$ conducted in the US or Canada, and a small number of studies $(n=$ 2) based on comparative data between high and low-/middleincome countries. The majority of studies ( $n=18$ ) focused on HIV vaccine trials, followed by vaginal microbicide trials $(n=6)$ (Table 1). 
In conducting thematic analysis, it became clear that the studies varied considerably in the extent to which they engaged with the contextual cultural specificities of trial sites. Furthermore, the studies reviewed had different stated objectives: some did not specifically aim to address or incorporate cultural context, while others expressly reported the objective of assessing the role of the cultural context in HIV prevention trials. All of the studies resulting from the search indicated the importance of addressing sociocultural considerations in trial activities; however, some contained little in the way of detail or tangible discussion of the unique contextual factors in the participating community. Importantly, the purpose of this review is not to evaluate individual studies; but we include all studies identified through our search strategy to illustrate a range of approaches to cultural sensitivity. In the themes described below, we discuss approaches that reflect the different levels of engagement with sociocultural considerations in order to more clearly distinguish among various approaches to engagement with culture in biomedical HIV prevention trials.

\section{Quality of reporting}

Quality of reporting, assessed with the 32-item COREQ, showed a range of items reported across studies (Table 2). All studies reported data on the number of participants and described major themes clearly. Nearly all studies (>90\%) included a description of audio/visual recording, derivation of key themes, demonstrative quotes, and appeared to demonstrate consistency between reported data and major findings. Most studies ( $<50 \%$ across all items) did not identify personal characteristics of the interviewer or focus group facilitator and did not discuss the relationship between the researcher and participants.

\section{Meta-synthesis}

We identified four analytic themes across the studies reviewed. Two of these themes reflected Bayer's conceptualization of cultural sensitivity: first, semantic issues relating to translating 
scientific terminology into the vernacular of target populations; and, second, issues relating to historical experience and mistrust that can and should be used instrumentally to tailor trial activities more appropriately. Two additional themes reflected challenges and considerations relating to the implementation and conceptualization of cultural sensitivity: first, budgetary, logistical, and personnel implications of operationalizing cultural sensitivity; and, second, cultural considerations as assets. Each of the themes demonstrates aspects of how cultural sensitivity is conceptualized and operationalized in biomedical HIV prevention trials in their formative, in-trial and/or post-trial activities.

\section{Semantic cultural sensitivity: linguistic and sociocultural meaning}

The first theme recognizes the importance of semantic cultural sensitivity by highlighting the challenges of rendering certain scientific concepts into the culturally relevant vernacular of particular communities. Semantic issues have been discussed extensively in studies of informed consent $[11,12,63,64]$ and were addressed in papers in the present meta-synthesis in relation to recruitment procedures [48,62] and communication/dissemination activities [39,61].

While several studies recognized the importance of 'cultural and linguistic competence' [37] in trial activities, some stopped short of outlining specific considerations for the trial community. A common approach was to search for the closest possible 'match' between scientific and vernacular terminology [61] or identify common concepts that caused confusion or misperceptions among trial communities, such as placebo, vaccine, randomization, or vaccineinduced seropositivity $[37,38,48,62]$. As one respondent in a US-based study explained, 'I think [clinical trials] are important, [but] that general information needs to be more user friendly. Language and jargon have the tendency to intimidate people' [45, p.255]. 
Several studies observed the need to go beyond finding equivalent meanings for study respondents. These studies frequently observed the importance of adapting data collection tools and communication methods to suit the sociocultural specificities of trial communities. In a study employing focus groups with diverse Latinos in Los Angeles to explore perceptions of HIV vaccine trials, the researchers note that merely translating English content into Spanish may elude culturally relevant concerns' [48, p.215]. A study of barriers and facilitators, to participation in HIV vaccine trials among transgender women included lengthy discussion of existing measurement tools and how these may be inappropriate for transgender individuals, particularly with regard to the representation of gender as a binary and the conflation of sex at birth with current gender identity.[35] Some studies advocated the use of visual technologies and media to assist in clarifying concepts and addressing semantic confusion, such as videos, slide presentations, and drawing pictures $[39,57]$ In a South African study, researchers hosted two weekly, $1 \mathrm{~h}$ phone-in talk shows on a local community radio station that addressed sexual and reproductive health questions, including HIV [39]. Another study developed a locally relevant analogy, 'one teabag is better than four' to explain to participants of a microbicide trial that 'a stronger concentration is not necessarily better than a weaker concentration at achieving a desired outcome' [41]. A study in India created a community-friendly PowerPoint presentation to explain scientific concepts in a 'simplistic, visual, and non-technical manner' during their meetings with potential volunteers [57, p.487].

Others undertook in-depth exploration of how scientific concepts were understood and interpreted by particular communities. Montgomery and colleagues [46, p.651] observe that 'cultural tailoring' of different biomedical terms and concepts 'may be required to achieve conceptual equivalence across different populations in different settings'. They explore the 'emic' interpretations of 'acceptability' in the context of a microbicide trial, observing the extent to which 'women's own experiences of gel use eclipse the categories currently proposed in the 
acceptability field' [46, p.651]. This 'incommensurability of fit' between biomedical concepts and participant perspectives should be explored in more detail across a variety of cultural contexts.

Similarly, in a study in India of 'mental models' that men who have sex with men (MSM) use to interpret scientific concepts, Chakrapani and colleagues [38, p.10] recommend going beyond the promotion of scientific literacy activities alone, to include an exploration of the 'underlying architecture of beliefs' to inform communication with trial participants. Importantly, as articulated in another study, semantic challenges can be compounded by historically based mistrust, with differing understandings of biomedical concepts becoming 'superimposed' on preexisting mistrust based on negative past encounters with medical research [52, p.12].

\section{Instrumental cultural sensitivity: historical experience and mistrust}

Many studies discussed the importance of understanding participants' experiences with trial research that may have fostered mistrustregarding the means or ends of clinical trials, and tailoring trial activities accordingly. Several studies contained references to participants feeling like they were being 'used as a guinea pig' [37-45,48,53,62]. In some instances, a common historical example was cited to explain mistrust of clinical research activities and advocate for greater cultural sensitivity. For example, a study exploring reasons for under-participation of African-Americans in HIV vaccine trials explained, 'Mistrust by blacks of medical research is due in part to the Tuskegee Study of Untreated Syphilis in the Negro Male (Tuskegee Syphilis Study), other sources may include the legacy of slavery' [45, p.254].

Though it is important to recognize historical antecedents to present-day distrust, undifferentiated reference to Tuskegee has been criticized for representing the study as a major cause of mistrust in the African-American community, rather than as 'a form of confirmation of what is already known or speculated about African-American treatment in medical systems' [65, 
p.955]. As such, citing the Tuskegee Syphilis Study can be a reflexive yet, at times, superficial act, perhaps detracting from a more in-depth analysis of the factors affecting participation in clinical research. McCallum and colleagues [66, p.730] conclude their study on the legacy of Tuskegee by observing, 'although minorities may generally mistrust medical research, are aware of the USPHS Syphilis Study at Tuskegee, and express an unwillingness to participate in medical research, they may participate at the same rate as Whites if recruited to participate'. As such, some argue that 'it is time we move beyond Tuskegee as a catch-allfor why African Americans mistrust medical care and begin to address the root causes' [65, p.956].

A similar phenomenon is demonstrated in some scholarship on mistrust of HIV prevention or treatment technologies in South Africa [42], whereby it is argued that the historical experience of apartheid 'still remains deeply present to many South Africans and explains much of the mistrust towards Western science, medicine and public health' [67, p.497]. Yet as with Tuskegee, citing the experience of apartheid has become a somewhat reflexive allusion to explain public distrust of biomedical HIV science.[68] Without detailed exploration of the specific roots or expressions of distrust, such explanations do little to reveal how experience informs current assessments of the trustworthiness of medical institutions. On the contrary, cursory descriptions of mistrust may support the misperception that deeply negative historical experiences can easily be overcome during a trial. A study of volunteers' experiences in a phase I/II HIV vaccine trial in Tanzania appears to confer this viewpoint by suggesting that 'the mistrust that we witnessed in this study can be minimized by timely dissemination of the trial information to the respective stakeholders' [60, p.11].

In a study designed to understand the reactions of rural Ugandans to preventive HIV vaccines, historical concerns raised by respondents are noted but appear to be minimally engaged with. Some respondents are reported to have serious concerns about the safety of vaccines, whereby 
'several interviewees referred to a specific past event where several people had died after vaccination, evidently because a vaccine was administered after its expiration date' [54, p.13]. In discussing these findings, the authors appear to underplay such concerns as resulting from 'rural cultures, where news frequently travels by word-of-mouth'. They argue, 'it is reasonable to expect adverse events to have enduring emotional impact and to be recalled with only partial accuracy' [54, p.15]. A study exploring factors that may enable or inhibit future South African HIV vaccine trials refers to rumors of a vaccine 'allegedly' leading to death and trial investigators who 'apparently' took no responsibility [44, p.754], which may confer a degree of skepticism about participants' accounts.

In contrast, several studies engaged in detailed discussion of the sociocultural context of the trial site that predated the commencement of trial-related research. A study of beliefs and perceptions of HIV vaccines by potential participants and community members in India highlighted fears that trials targeted economically disadvantaged people from developing countries.[38] A community member asked for specific clarification on the selection criteria for study sites and populations, seeking reassurance that other countries and communities were also being included. The authors explore the possibility that these concerns may be particularly salient for MSM communities in India because of their disenfranchisement from mainstream Indian society, and recommend 'explicitly addressing doubts in the minds of both MSM community leaders and grassroots level MSM about the motivations behind HIV vaccine trials' [38,p.9].

A study of community preparedness for HIV vaccine trials in the Democratic Republic of Congo similarly highlighted the importance of establishing trust in vaccine campaigns in the face of two specific historical controversies that may impact on clinical trials of HIV prevention technologies [53]. The first, polio vaccine research conducted in the 1950s in Eastern Congo using a vaccine 
from African green monkey cell cultures, fuelled beliefs that AIDS was manufactured by researchers. Second, an early HIV vaccine trial in Kinshasa used children as research subjects and investigators were accused of engaging in unethical conduct. The authors conclude, 'such cultural and historical biases must be overcome to build understanding about and trust in clinical trials' [53, p.542].

Similarly, in clinical trials evaluating treatment for herpes simplex virus infection as a strategy for HIV prevention, the authors note how the historical context of the study country - South Africa was incorporated and addressed throughout the process of administering the trials and communicating the study results [39]. Rather than a cursory allusion to the experience of apartheid, this paper draws clear links between their communication activities in the present and the sociocultural context that underlies their involvement in the trial communities. First, they describe the distrust of research activities that is one of the legacies of apartheid, rooted both in widespread reports that in the final years of the apartheid regime the government was collaborating with scientists to spread HIV in the black population, as well as more contemporary debates in South African society about the origins of AIDS. Second, they discuss another legacy of apartheid in the form of the skills shortage, specifically the low number of personnel at the local, provincial and national levels who have the abilities to draw on scientific findings to inform policy. In response, the authors describe how they, as researchers, were 'frequently drawn into mediating these tensions between stakeholders, rather than playing the more traditional role of a "neutral observer"' [39, p.2].

Significant consideration was given to the historical context in a study embedded within a vaginal microbicide trial in South Africa, in which several respondents expressed concerns that the trial might harm participants. Contextualizing these observations, the authors remark, 'Marshaling evidence to corroborate these accusations, some participants and residents 
remarked that other microbicide trials had also put their participants at risk. Many had seen medical reports regarding the Zambian Cellulose Sulphate trial, which was suspended after higher rates of HIV infection appeared among those using the product as compared to those randomized to the placebo' $[55$, p.106]. By rooting participants' concerns in tangible observations about experiences with biomedical trials, the authors take culture seriously, imparting the specific sociocultural context of the trial site and drawing clear links between respondents' observations about past events and their current interpretations of research activities.

\section{Logistical, budgetary, and personnel implications}

A third theme that emerged from the meta-synthesis was the tangible implications - logistical, budgetary, and personnel - of addressing contextual specificities of potential or actual HIV prevention trial participants. Some studies described the need for specific recruitment and engagement strategies that reflect the unique cultural considerations of the community in question. In their discussion of strategies for recruiting the steady male partners of female sex workers, Fleming and colleagues [40] recommended using data collection methods that 'are designed to make participants feel more comfortable sharing personal details', particularly in cultures where men 'are socialized to limit their expression of emotions in front of other people'. A community-based recruitment strategy in India was similarly 'labor intensive'; researchers undertook a three-step approach, whereby information was shared with the community, trialists received inputs from community representatives about salient local norms, values and priorities, and trialists then incorporated these inputs into the educational programs designed to communicate about the trial activities to potential participants [57]. The investigators also describe specific measures to facilitate recruitment of women, including sensitivity training for all staff, attention among recruiters to the importance of spouses and families, and the provision of free childcare [57]. 
Researchers describe a number of strategies employed in preparation for a study evaluating intravaginal rings as a potential HIV prevention device for sex workers and their male clients in Mukuru, an informal settlement in Nairobi, Kenya. They held community meetings with local administrators and village elders, as well as mapping Mukuru to identify bars, brothels, and other locations where female sex workers and their clients could be reached [58]. Ina study of perspectives on HIV vaccine trials among homeless youth in Los Angeles, the researchers caution that 'young adults in transition or without stable social environments require special considerations with regard to access and follow-up points, flexible scheduling for data collection, and the use of incentives [43]. Context-specific logistical concerns were similarly described in a study of HIV vaccine trial participation among high-risk women in Philadelphia, in which participants voiced concerns about factors in their daily lives that would make it difficult to commit to regular appointments; the authors suggest 'providing rides to office visits when necessary, and/or conducting clinical study visits in more accessible neighborhood locations' [62].

Investigators also described significant commitments arising from having to 'step outside' of their traditional role as researchers $[39$, p.6] in order to actively engage in a culturally sensitive manner with community members.

In the case of a study about the processes of communicating results from an HSV/HIV intervention in South Africa, the authors highlighted the myriad activities they undertook to ensure that the study and its findings were understood by community members, including extensive training for trial staff in communication and media handling, radio shows, meetings with government officials, text messages, community workshops, and home visits [39]. The resources for these diverse dissemination activities came from the trial, at considerable cost, and 'remain a concern for investigators generally' $[39, p .8]$. The researchers also expressed 
concern about the allocation of time for these activities; though worthwhile and key to 'creating a more enabling environment for future research to take place', they also distract from 'the academic expectations that they publish' [39, p.8]. Though these researchers generally lack budgets that enable them to address wider social and economic inequalities in study communities, when possible, some described using their leverage with service delivery organizations to address some of the wider needs of the participating community.[39] This example illustrates personnel, logistical, and budgetary implications of incorporating culturally sensitive approaches in a trial, as well as highlighting how even well-funded trials may experience limitations on their ability to address broader social dynamics in trial sites.

A similarly comprehensive approach to incorporating cultural specificities was described in a research partnership formed with leaders from the House Ball Committee, a subset of the lesbian, gay, bisexual, and transgender (LGBT) community of color primarily composed of young black gay and bisexual men, and centered on creative expression, dance, and music.[34] Drawing on principles of communitybased participatory research, the partnership aimed to improve knowledge of HIV prevention and HIV vaccine trial participation. One of the study interventions was anCevent whereby House Ball community members were encouraged to perform, compete, and express themselves creatively while also affirming the use of safer sex practices and reducing stigma associated with participation in clinical trials. In a study with black MSM, Andrasik and colleagues [36] describe the importance to culturally relevant and community informed research of engaging in true partnerships with community research partners, including engagement of community partners as coinvestigators on grant applications, as research consultants, and coauthors on publications, all with the aim of increasing the capacity of community-based organizations to conduct research. They describe a two-way process of cultural sensitivity: 'By working toward a mutually beneficial and equitable 
relationship, community members can improve their research literacy, while researchers enhance their cultural awareness, responsiveness, and sensitivity to the community' [36, p.713].

Other studies identified the importance of 'going beyond' what is traditionally included in clinical trial activities - structured questionnaires, biomarkers, etc. to include more participatory methods. In a study of barriers and facilitators to participation of transgender women in HIV vaccine trials, the authors highlight the potential benefits of mixed method researchin helping researchers develop 'more transfriendly and participant-focused clinical trials' [35, p.273]. An HIV vaccine trial in India applied participatory action research to facilitate recruitment, including active involvement of a Community Advisory Board and the media, capacitation of communitybased volunteers to provide trial information, and a public presentation tailored to the needs and interests of the community.[57]

A vaginal microbicide trial in South Africa demonstrated high cultural sensitivity in addressing and incorporating the 'emic' or 'insider' perspective from the outset of the trial.[46,55-70] Concern for cultural specificities of the trial community was operationalized by embedding social scientists in the research team early in the trial design in order to better 'assess the impact of trials on the community as well as inform current and future clinical trials in Johannesburg' [56, p.100]. By employing a mixed methods approach, including in-depth semistructured interviews, focus group discussions, and participant observation,[59] the trial team indicated its commitment to gaining 'a broader knowledge of rumors and awareness of the trial, vaginal cleansing practices, health and healing, sexual partnerships and HIVIAIDS' [55, p.105]. The qualitative component aimed to 'assess and contribute to increasing the accuracy of these data through the use of a multi-method data collection strategy and the triangulation of results' [69, p.66]. Treating social scientists as 'an official and integral component' [55, p.105] of a trial remains a rarity and requires a significant allocation of personnel, time, and financial support 
above and beyond basic requirements for the clinical investigation. Yet as the members of the social science component of the trial note, this should be considered money and time well spent. If integrating social scientists and using mixed methods to triangulate findings 'is not done then trialists risk having spent millions of dollars and still ending up not knowing what it was they paid so much to find out' $[70, p .8]$.

\section{Culture as an asset}

Finally, in discussing the specific cultural considerations of each trial community, some studies focused exclusively on aspects of the sociocultural context that pose challenges to the implementation of bio-medical HIV prevention trials. For instance, an investigation of attitudes and behaviors related to intravaginal rings as potential HIV prevention devices in Kenya explored 'cultural practices' in order to provide 'important social and cultural context' in their responses, but reported only those that hindered use of the intravaginal ring and/or increased susceptibility to HIV.[58]

In other studies, researchers recognized that characteristics of each sociocultural context could be framed as assets rather than viewing all cultural phenomena as challenges. For instance, in a Los Angeles-based study reporting 'some Latino/a cultural norms, if they are not appropriately engaged and addressed, may present barriers to participation in HIV vaccine trials' [37, p.215], the investigators also draw attention to customs and values that could be harnessed to strengthen and support biomedical HIV prevention trials. The benefits expressed by participants of providing free insurance and medical care were linked to the importance placed on extended family in Latino communities as a way of ensuring that other family members would be cared for regardless of the health status of the participant.[48] A study of HIV vaccine acceptability in Thailand reported that Buddhist values and communitarian norms in Thai culture served as 
cultural assets in the promotion of HIV vaccines: 'A social vaccine is very important; the family, the friends, the community need to support them [vaccine recipients]' [51, p.5].

Another example of cultural norms being framed as assets is in Alio and colleagues' [34] research partnership with the House Ball Committee. In their description of the House Ball subculture, the authors highlight several advantages: it is tightly knit, stable, and hierarchical, constituting 'an important asset that must be accessed if HIV prevention and recruitment for participation of minority community members in HIV vaccine trials is to be improved' [34, p.10]. House Ball Committee leaders have a great deal of influence on members, who are often displaced LGBT youth seeking guidance and support. Though socially and economically marginalized, the House Ball Committee, therefore, provides an excellent infrastructure in which to conduct HIV interventions and HIV vaccine educational programs' [34, p.10].

In each of these studies, sociocultural specificities are viewed as complex and nuanced, with the potential to create challenges for the successful implementation of biomedical HIV prevention trials; but they are also approached as having the potential to serve as points of resilience or community strength for clinical trials to draw upon. Research teams endeavoring to conduct clinical trials with specific populations are well served to attune themselves to the assets and advantages offered by particular cultural values and practices, rather than focusing solely on cultural specificities as obstacles to trial implementation.

\section{Limitations}

Limitations to this meta-synthesis include the heterogeneity of studies reviewed, including their primary focus, samples, geographical location, and the degree of operationalization of cultural sensitivity. Despite these differences, we identified well-supported themes across 29 qualitative investigations. Many studies included in this meta-synthesis were conducted in the Global 
South, allowing for similar contexts of historical experiences of colonialism, ongoing racial/ ethnic tension, political instability, and poverty in countries that experience a disproportionate burden of the global HIV epidemic. Among the 10 studies primarily conducted in the US or Canada, study samples consisted of key populations who experience a disproportionate burden of HIV, as well as other health and economic disparities, stigma, and discrimination, such as ethnoracialized communities, trans women, and gay and other MSM.

\section{Expert commentary}

This meta-synthesis focused on qualitative studies that address processes of adapting formative or in-process activities for biomedical HIV prevention trials to the cultural specificities of trial populations. Overall, few studies focused on interventions conducted to address sociocultural complexities and nuances of particular populations. More common are studies exploring 'facilitators and barriers' to participation in ongoing or future HIV prevention trials or designed to improve participation rates among historically under-represented populations in medical research. These may yield important insights at a formative stage that can be implemented in future trial activities; however, accounts of the actual, tangible operationalization of sociocultural considerations in trial preparedness, implementation, and dissemination activities are crucial.

This synthesis of existing research reveals, first, a relative surfeit of studies focused on tailoring informed consent processes to local cultural specificities as compared to the few studies focused on other aspects of formative and in-process trial activities. This may speak to the perception of informed consent as the one aspect of clinical trials that must immutably be aligned with participants' cultural and linguistic norms. Yet as this meta-synthesis suggests, there are numerous opportunities to address the mandate of semantic and instrumental cultural sensitivity throughout the course of a trial: in preparedness efforts and at the outset, when historical experiences of medical research within participating communities can be 
explored and trial activities tailored accordingly; during the trial, when participation and input from communities can be incorporated; and after a trial has been completed, when dissemination of results can be tailored to suit contextual specificities. Reasons for the relative neglect in published manuscripts of how semantic and instrumental cultural sensitivity is applied in these other aspects and stages of trial implementation should be explored.

Second, the extensive nature of some of the strategies employed in clinical trials to engage with sociocultural contexts highlights the budgetary, logistical, and personnel-costs associated with undertaking a comprehensively contextualized clinical trial. As some investigators note, the resource implications of conducting wide-ranging formative activities prior to a trial, having a cadre of social scientists embedded in a trial team, or deploying creative strategies for dissemination activities are significant, both in terms of what is required and allocated in a trial budget and in relation to the time and personnel (and skill sets) required to implement these activities appropriately. The good intentions of trial teams to tailor activities to the cultural specificities of particular communities are a necessary but not sufficient condition for the fulfillment of these goals; appropriate allocation of time, money, and personnel resources is essential. If one considers the resources squandered due to international trial shutdowns that may occur in part as a result of neglect of cultural considerations, such investment may prove cost effective in the longer term.

Third, this meta-synthesis represents a range of studies that demonstrate a broad spectrum of conceptualizations and operationalizations of 'cultural sensitivity' in clinical trials. Some studies merely referred to the importance of sociocultural sensitivity in trial activities, largely absent descriptive or conceptual detail; other studies explored in-depth the manner in which sociocultural specificities of a trial community might be addressed. Importantly, a few studies were notable in adopting a broad and adaptive view by approaching cultural characteristics not 
only as challenges to be addressed but as assets to be promoted in launching HIV prevention trials.

Finally, the heterogeneity of the studies reviewed is notable and may usefully point to different approaches that can be employed to operationalize cultural sensitivity depending on one's research objectives. Some studies utilized an understanding of culture applied to a disenfranchised or vulnerable population, such as transgender persons, [35] while others assessed culture through the lens of nation/country,[39] and still others assessed culture from a combination of disenfranchised population and nation/country lenses.[51] Some studies focused on trial preparation,[53] while others focused on ongoing trial activities,[56] and others focused on trial closure and dissemination of results,[39] reflecting the breadth of strategies that may be needed to meaningfully operationalize cultural sensitivity in clinical trials.

\section{Five-year view}

Importantly, this review suggests the need for increased focus on cultural sensitivity and how it is conceptualized and operationalized across the spectrum of biomedical HIV prevention trials. This includes trials conducted in resource-limited settings and trials conducted with disenfranchised populations in high-, middle-, and low-income settings. Preparedness studies and biomedical prevention trials in the next five years should aspire to the standards set, and methods described, in those investigations that demonstrated a high value placed on in-depth, comprehensive operationalization and analyses of cultural sensitivity, and that address culture as an asset in addition to a challenge.

Several important considerations that were beyond the scope of the studies reviewed suggest directions for future research. For one, this meta-synthesis indicates the importance of investigations that specifically address how sociocultural considerations are operationalized and 
integrated in particular activities across the spectrum of trial preparedness, implementation, and dissemination of results, and in particular sociocultural contexts. Another crucial direction for future studies is to tangibly evaluate the effectiveness of the methods used to integrate sociocultural considerations at multiple points in the research process. For example, investigations might assess trial participants' perceptions of trust after an intervention to address historical experiences in preparation for a trial, participant engagement after an intervention to enhance sociocultural appropriateness of data collection methods, or community understanding of trial results after an intervention to enhance sociocultural appropriateness of dissemination. Intervention studies that incorporate evaluation components will provide much needed evidence to guide future trial activities that aim to effectively operationalize culturally sensitive practices.

\section{Financial \& competing interests disclosure}

PA Newman is principal investigator on studies funded by the Canadian Institutes of Health Research (THA-118570), and receives funding from the Canada Research Chairs Program. The authors have no other relevant affiliations or financial involvement with any organization or entity with a financial interest or financial conflict with the subject matter or materials discussed in the manuscript apart from those disclosed.

\section{Key issues}

- Approaches to conceptualizing and operationalizing cultural sensitivity in biomedical HIV prevention trials range from superficial consideration (e.g. language as culture) to in-depth implementation activities and nuanced analyses (e.g. incorporating historical and colonial contexts, and perspectives from participants' firsthand experiences of the trial).

- Semantic challenges arise in communicating biomedical concepts to audiences with different understandings and vocabularies. 
- Trialists' understanding of how historical experiences of trial communities may be manifest in participants' responses to and interpretations of trial activities is important to enable meaningful tailoring of activities and content across the spectrum of trial preparedness, implementation, and dissemination.

- Logistical, budgetary, and personnel implications of undertaking activities to meaningfully operationalize cultural sensitivity throughout the lifecycle of a trial must be considered, and such activities appropriately resourced.

- Researchers should highlight and approach sociocultural specificities as assets as well as challenges in preparedness and implementation of successful biomedical HIV prevention trials.

- Preparedness studies and biomedical prevention trials in the next five years should aspire to the standards set, and methods described, in the few investigations highlighted that demonstrate a high value placed on in-depth, comprehensive operationalization and analyses of cultural sensitivity. 


\section{References}

Papers of special note have been highlighted as:

- of interest

•• of considerable interest

1. Newman PA. Towards a science of community engagement. Lancet. 2006;367:302.

2. Tindana PO, Singh JA, Tracy CS, et al. Grant challenges in global health: community engagement in research in developing countries. PLoS Med. 2007;4(9):e273.

- Summarizes key issues relating to community engagement, including definitions of 'community' and 'engagement', conceptual models and examples of successful practices

3. Marsh V, Kamuya D, Rowa $\mathrm{Y}$, et al. Beginning community engagement as a busy biomedical research programme: experiences from the KEMRI CGMRC-Wellcome Trust Research Programme, Kilifi, Kenya. Sog Sci Med. 2008;67 (5):721-733.

4. Lavery JV, Tinadana PO, Scott TW, etal. Towards a framework for community engagement in global health research. Trends Parasitol. 2010;26(6):279-283.

- Addresses some of the gaps in current guidance around community engagement processes in global research and highlights pressing ethical and practical questions related to the inclusion of socioculltural values, norms, and preferences

5. Participants in the 2001 Conference on Ethical Aspects of Research in Developing Countries. Moral standards for research in developing countries from "reasonable availability" to "fair benefits.". Hastings Cent Rep. 2004;34 (3):17-27.

- Articulates the interdependencies between informed consent and community engagement, drawing on the authors' extensive field experience to illustrate challenges, strategies and suggestions for further social science research

6. UNAIDS-AVAC. Good participatory practice: guidelines for biomedical HIV prevention trials. Geneva (Switzerland): UNAIDS; 2007. 
7. UNAIDS-AVAC. Good participatory practice: guidelines for biomedical HIV prevention trials. 2nd ed. Geneva (Switzerland): UNAIDS; 2011.

•• Establishes foundational values and priorities to guide biomedical HIV prevention trials

8. Nuffield Council on Bioethics. The ethics of research related to healthcare in developing countries. 2002. [cited 2015 Aug 28]. Available from: http://nuffield bioethics.org/wpcontent/uploads/2014/07/Ethics-of-research-related-to-healthcare-indeveloping-countries-I. pdf

9. National Bioethics Advisory Commission. Ethical and policy issues in international research: clinical trials in developing countries. Volume 1: report and recommendations of the National Bioethics Advisory Commission. 2001. [cited 2015 Aug 28] Available from: https://bioethicsarc hive.georgetown.edu/nbac/clinical/Vol1.pdf

10. AVAC. GPP Blueprint for stakeholder engagement. 2014. [cited 2015 Aug 28]. Available from: http://www.avac. org/resource/gpp-blueprint-stakeholder-engagement.

- Provides tangible guidance on how to implement stakeholder engagement activities in biomedical HIV prevention research

11. Corneli AL, Bentley ME, Sorenson JR, et al. Using formative research to develop a contextspecific approach to informed consent for clinical trials. J Empir Res Hum Res Ethics. 2006;1(4):45-60.

12. Watermeyerd, Renn J. "They take positive people": an investigation of communication in the informed consent process of an HIV/AIDS vaccine trial in South Africa. Crit Inq Lang Stud. 2008;5(2):81-108.

13. Gikonyo C, Bejon P, Marsh V, et al. Taking social relationships seriously: lessons learned from the informed consent practices of a vaccine trial on the Kenyan Coast. Soc Sci Med. 2008;67(5):708-720. 
14. Lindegger G, Milford C, Slack C, et al. Beyond the checklist: assessing understanding for HIV vaccine trial participation in South Africa. J Acquir Immune Defic Syndr. 2006;43(5):560-566.

15. Woodsong $\mathrm{C}$, Alleman $\mathrm{P}$, Musara $\mathrm{P}$, et al. Preventive misconception as a motivation for participation and adherence in microbicide trials: evidence from female participants male partners in Malawi and Zimbabwe. AIDS Behav. 2012;16:785-790.

16. Marshall PA. "Cultural competence" and informed consent in international health research. Camb Q Healthc Ethics. 2008;7(02):206-215.

17. Molyneux S, Mulupi S, Mbaabu L, et al. Benefits and payments for research participants: experiences and views from a research centre on the Kenyan Coast. BMC Med Ethics. 2012;13(1):13.

18. Staunton C. Informed consent for HIV cure research in South Africa: issues to consider. BMC Med Ethics. 2015;16 (1):3.

19. Lindegger G, Richter LM. HIV vaccine trials: critical issues in informed consent. S Afr J Sci. 2000;96:313-317.

20. Lindegger G, Bull S Ensuring valid consent in a developing country context. SciDev.Net. 2002. [cited 2015 Aug 28]. Available from: http://www.scidev.net/index.cfm? originalUrl=global/policy-brief/ensuring-valid-consent-ina-developing-country-con.html.

21. Bayer R. AIDS prevention and cultural sensitivity: are they compatible?". Am J Public Health 1994;84(6):895-898.

- Reflects on key conceptual questions relating to cultural sensitivity

22. Sandelowski M, Barroso J. Handbook for synthesizing qualitative research. New York (NY): Springer; 2007.

23. Limkakeng A, Phadtare A, Shah J, et al. Willingness to participate in clinical trials among patients of Chinese heritage: a meta-synthesis. PLoS One. 2013;8(1):e51328. 
24. Abbott LS, Williams CL. Influences of social determinants of health on African Americans living with HIV in the rural southeast: a qualitative meta-synthesis. J Assoc Nurses AIDS Care. 2015;26(4):340.

25. Tuthill E, McGrath J, Young S. Commonalities and differences in infant feeding attitudes and practices in the context of HIV in sub-Saharan Africa: a metasynthesis. AIDS Care. 2014;26(2):214-225.

26. Sandelowski M, Barroso J. Toward a metasynthesis of qualitative findings on motherhood in HIV-positive women. Res Nurs Health. 2003;26(2):153-170.

27. Toye F, Seers K, Allcock N, et al. Meta-ethnography 25 years on: challenges and insights for synthesising a large number of qualitative studies. BMC Med Res Methodol. 2014;14(1):80.

28. Greenhalgh T, Peacock R. Effectiveness and efficiency of search methods in systematic reviews of complex evidence: audit of primary sources. BMJ. 2005;331 (7524):1064-1065.

29. Moher D, Liberati A, Tetzlaff J, et al. Preferred reporting items for systematic reviews and meta-analyses: the PRISMA statement. PLoS Med. 2009;6(7):e1000097.

30. Tong A, Sainsbury P, Craig J. Consolidated criteria for reporting qualitative research (COREQ): a 32-item checklist for interviews and focus groups. Int $\mathrm{J} Q$ Health Care. $2007 ; 19(6): 349-357$.

31. Thomas J, Harden A. Methods for the thematic synthesis of qualitative research in systematic reviews. BMC Med Res Methodol. 2008;8:45.

32. Noblit GW, Hare DR. Meta-ethnography: synthesizing qualitative studies. Newbury Park (CA): Sage; 1988.

33. Patterson B, Thorne S, Canam C, et al. Meta-study of qualitative health research: a practical guide to meta-analysis and meta-synthesis. Thousand Oaks (CA): Sage; 2001. 
34. Alio AP, Fields SD, Humes DL, et al. Project VOGUE: a partnership for increasing HIV knowledge and HIV vaccine trial awareness among house ball leaders in western New York. J Gay Lesbian Soc Serv. 2014;26(3):336-354.

- Provides in-depth examination of the steps required to engage in collaborative research partnerships with key knowledge users and the use of innovative strategies that are socioculturally specific to the target community

35. Andrasik MP, Yoon R, Mooney J, et al. Exploring barriers and facilitators to participation of male-to-female transgender persons in preventive HIV vaccine clinical trials. Prev Sci. 2014;15(3):268-276.

36. Andrasik MP, Chandler C, Powell B, et al. Bridging the divide: HIV prevention research and black men who have sex with men. Am J Public Health 2014;104(4):708-714.

37. Brooks RA, Newman PA, Duan N, et al. HIV vaccine trial preparedness among Spanishspeaking Latinos in the US. AIDS Care. 2007;19(1):52-58.

38. Chakrapani V, Newman PA, Singhal Net al. "If it's not working, why would they be testing it?: mental models of HIV vaccine trials and preventive misconception among men who have sex with men in India. BMC Public Health. 2013;13(1):731.

39. Delany-Moretlwe S, Stadler J, Mayaud P, et al. Investing in the future: lessons learnt from communicating the results of HSV/HIV intervention trials in South Africa. Health Res Policy Sys. $2011: 9(\mathrm{~S}): 1-9$.

- Highlights the necessity for researchers to 'step outside' of their traditional clinical roles and engage with the wider contextual environment in which the trial takes place

40. Fleming PJ, Barrington C, Perez M, et al. Strategies for recruiting steady male partners of female sex workers for HIV research. AIDS Behav. 2015;19(2):362-368.

41. Gafos M, Mzimela M, Ndlovu H, et al. "One teabag is better than four": participants response to the discontinuation of $2 \%$ PRO2000/5 microbicide gel in KwaZuluNatal, South Africa. PLoS One. 2011;6(1):e14577. 
42. Jaspan HB, Soka NF, Mathews C, et al. A qualitative assessment of perspectives on the inclusion of adolescents in HIV vaccine trials in South Africa. Int J STD AIDS. 2010;21(3):172-176.

43. Koniak-Griffin D, Nyamathi A, Tallen L, et al. Breaking the silence: what homeless 18to 24year-olds say about HIV vaccine trials. J Health Care Poor Underserved. 2007;18 (3):687698.

44. Lesch AM, Kafaar Z, Kagee A, et al. Community members' perceptions of enablers and inhibitors to participation in HIV vaccine trials. S Afr J Psych. 2006;36(4):734-761.

45. Moutsiakis DL, Chin PN. Why blacks do not take part in HIV vaccine trials.". J Nat Med Assoc. 2007;99(3):254-257.

46. Montgomery CM, Gafos M, Lees S, et al. Re-framing microbicide acceptability: findings from the MDP301 trial. Cult Health Sex. 2010,12(6):649-662.

47. Morrow K, Rosen R, Richter L, et al. The acceptability of an investigational vaginal microbicide, pro 2000 gel, among women in a Phase I clinical trial. J Women's Health. 2003;12(7):655-666.

48. Newman PA, Duan N, Roberts KJ, et al. HIV vaccine trial participation among ethnic minority communities Cbarriers, motivators, and implications for recruitment. J Acquir Immune Defic Syndr. 2006;41(2):210-217.

49. Newman PA, Logie C, James L, et al. "Speaking the dialect": understanding public discourse in the aftermath of an HIV vaccine trial shutdown. Am J Public Health. 2011;101(9):1749-1758.

50. Newman PA, Yim S, Daley A, et al. "Once bitten, twice shy": participant perspectives in the aftermath of an early HIV vaccine trial termination. Vaccine. 2011;29(3):451-458.

51. Newman PA, Roungprakhon S, Tepjan S, et al. A social vaccine? Social and structural contexts of HIV vaccine acceptability among most-at-risk populations in Thailand. Glob Public Health. 2012;7(9):1009-1024. 
52. Newman PA, Rubincam C, Slack C, et al. Towards a science of community stakeholder engagement in biomedical HIV prevention trials: an embedded four-country case study. PLoS One. 2015;10(8):e0135937.

- Articulates challenges that arise around trial literacy, historical mistrust, and community stakeholder engagement across four country settings of HIV prevention trials, and gaps between avowed best practices and their implementation

53. Olin J, Kokolamami J, Lepira FB, et al. Community preparedness for HIV vaccinetrials in the Democratic Republic of Congo. Cult Health Sex. 2006;8(6):529-544.

54. Ritvo P, Willms D, Meisner R, et al. Vaccine preparedness: lessons from Lyantonde, Uganda. Afr J AIDS Res. 2006;5 (1):11-16.

55. Saethre E, Stadler J. Malicious whites, greedy women, and virtuous volunteers. Med Anthropol Q. 2013;27 (1):103-120.

56. Saethre E, Stadler J. Gelling medical knowledge: innovative pharmaceuticals, experience, and perceptions of efficacy. Anthropol Med. 2010;17(1):99-111.

- Provides insights from a large clinical trial that had social scientists embedded in the research team from the outset, revealing depth of understanding from participants' experiential perspectives and substantial disjoints between participant and researcher conceptions of the trial

57. Sahay S, Kamar-M, Srikrishnan AK, et al. Experiences in recruiting volunteers through community based initiatives in phase-1 vaccine trials in India. Hum Vaccin Immunother. 2014;10(2):485-491.

58. Smith DJ, Wakasiaka S, Hoang TDM, et al. An evaluation of intravaginal rings as a potential HIV prevention device in urban Kenya: behaviors and attitudes that might influence uptake within a high-risk population. J Women's Health. 2008;17(6):1025-1034.

59. Stadler J, Saethre E. Rumours about blood and reimbursements in a microbicide gel trial. Afr J AIDS Res. 2010;9 (4):345-353. 
60. Tarimo EAM, Thorson A, Kohi TW, et al. A qualitative evaluation of volunteers' experiences in a phase I/II HIV vaccine trial in Tanzania. BMC Infect Dis. 2011;11(1):283.

61. Van Der Elst EM, Mbogua J, Operario D, et al. High acceptability of HIV pre-exposure prophylaxis but challenges in adherence and use: qualitative insights from a phase I trial of intermittent and daily PrEP in at-risk populations in Kenya. AIDS Behav. 2013;17(6):21622172.

62. Voytek CD, Jones KT, Metzger DS. Selectively willing and conditionally able: HIV vaccine trial participation among women at 'high risk' of HIV infection. Vaccine. 2011;29 (36):6130-6135.

63. Corbie-Smith G, Thomas SB, Williams MV, et al. Attitudes and beliefs of African Americans toward participation in medical research. J Gen Intern Med. 1999;14(9):537-546.

64. Rautenbach C, Lindegger L, Slack C, et al. l'm positive, but I'm negative: competing voices in informed consent and implications for HIV vaccine trials. J Empir Res Hum Res Ethics. 2015;10(2):151-156.

65. Brandon DT, Isaac LA, LaVeist TA. The legacy of Tuskegee and trust in medical care: is Tuskegee responsible for race differences in mistrust of medical care? J Nat Med Assoc. 2005;97(7):951-956.

66. McCallum JM, Arekere DM, Green GL, et al. Awareness and knowledge of the U.S. public health service syphilis study at Tuskegee: implications for biomedical research. J Health Care Poor Underserved. 2006;17(4):716-733.

67. Fassin D, Schneider H. The politics of AIDS in South Africa: beyond the controversies. BMJ. 2003;326:495-497.

68. Rubincam C. Alternative beliefs about HIV and AIDS: reexamining distrust among young adults in Cape Town, South Africa. Centre for Social Science Research, University of Cape Town. 2014. [cited 2015 Aug 28]. Available from: http://www.cssr.uct.ac.za/pub/wp/342. 
69. Pool R, MDP team. Some issues arising from the use of qualitative methods in clinical trials. In: Proceedings of the 2007 KNAW Colloquium, 63-75; Amsterdam (Netherlands): Johannes van Kessel; 2007.

70. Pool R, Montgomery CM, Morar NS, et al. A mixed methods and triangulation model for increasing the accuracy of adherence and sexual behaviour data: the microbicides development programme. PLoS One. 2010;5(7): e11600.

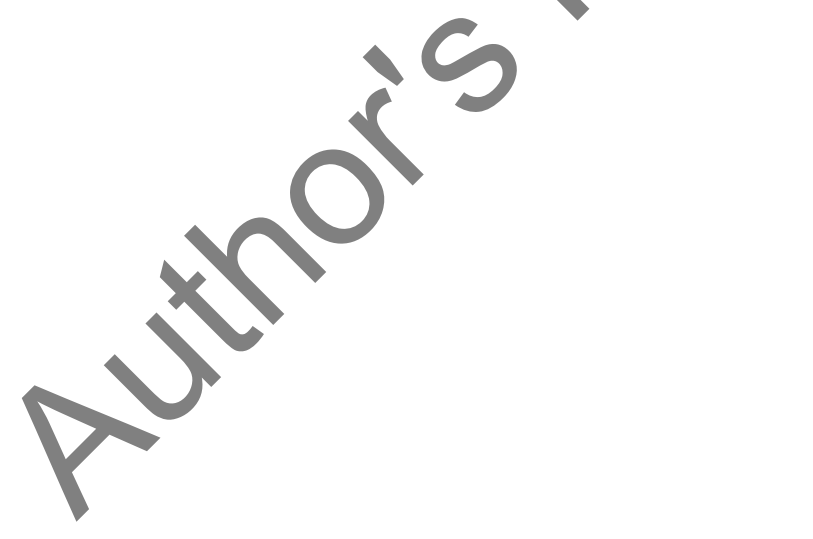


Figure 1. Flow diagram of study selection

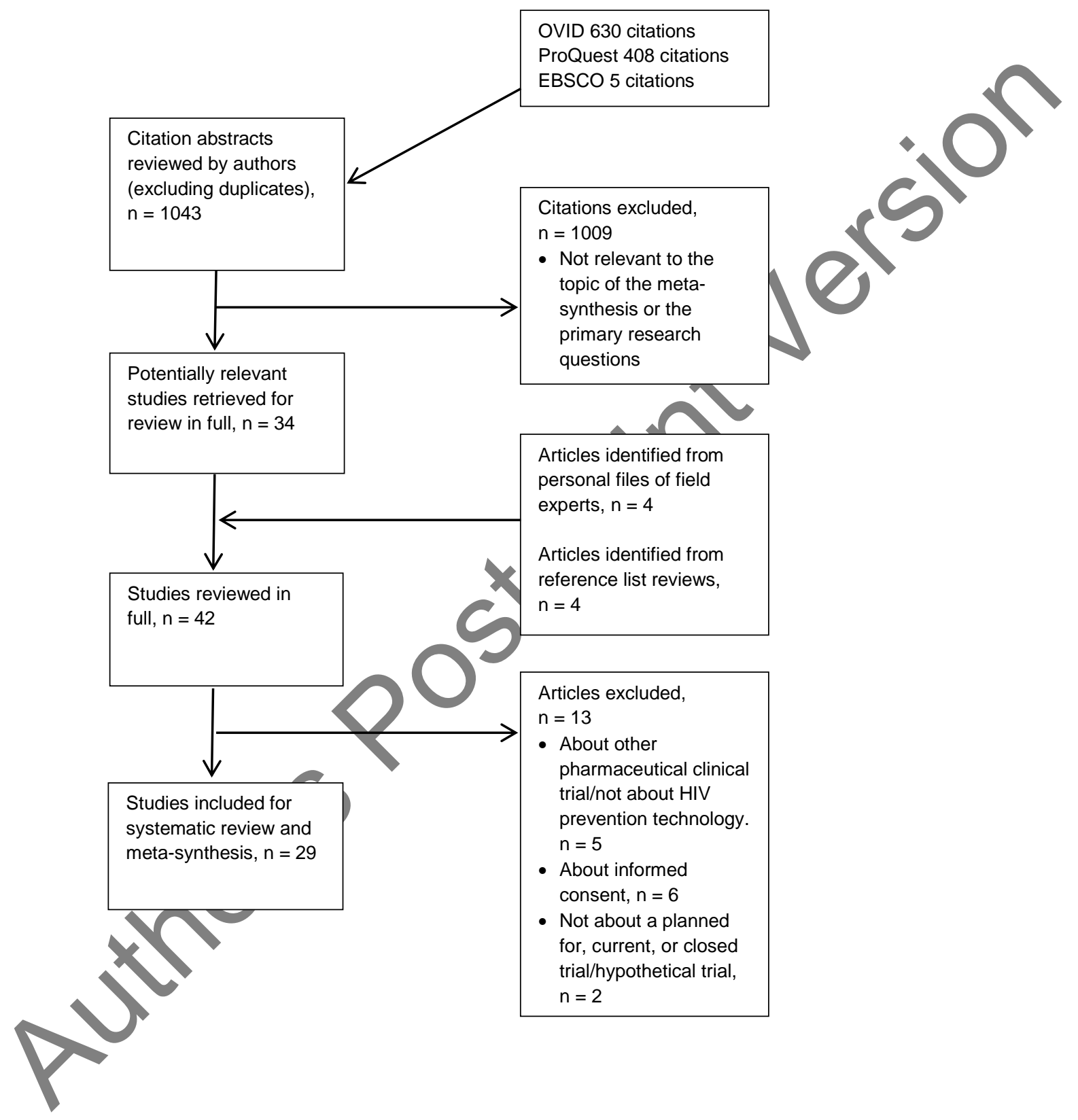


Table 1. Characteristics of included studies, $n=29$

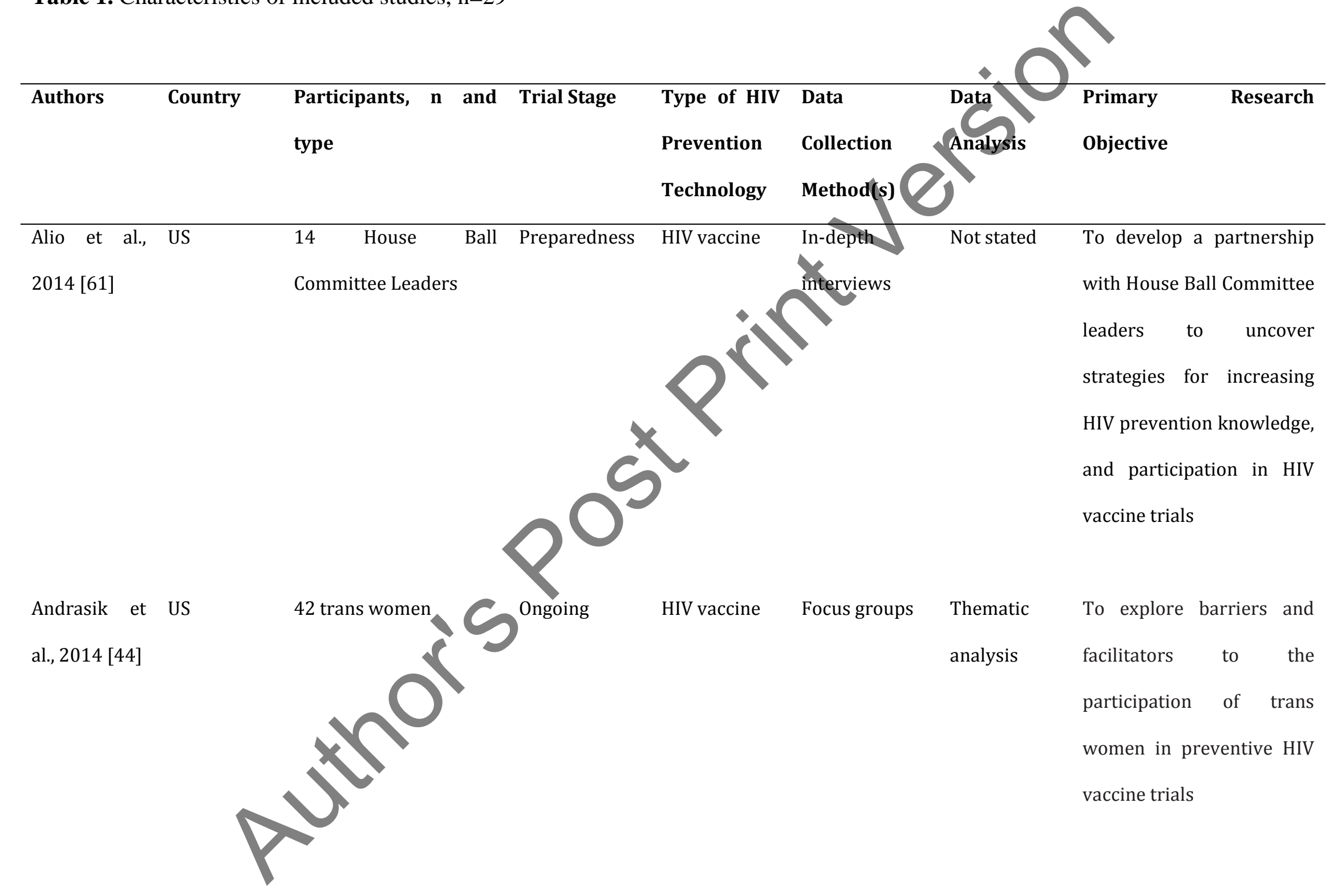




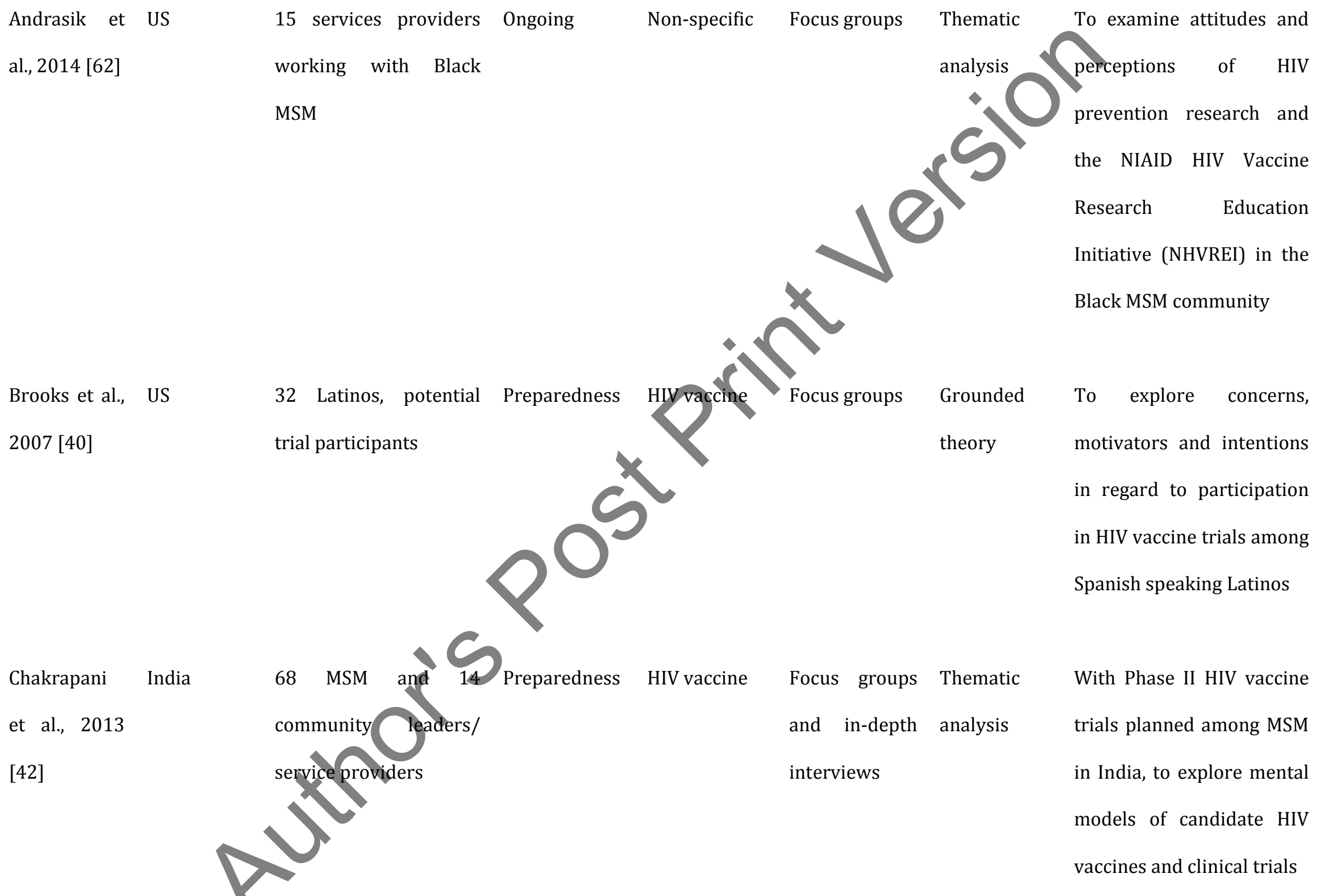




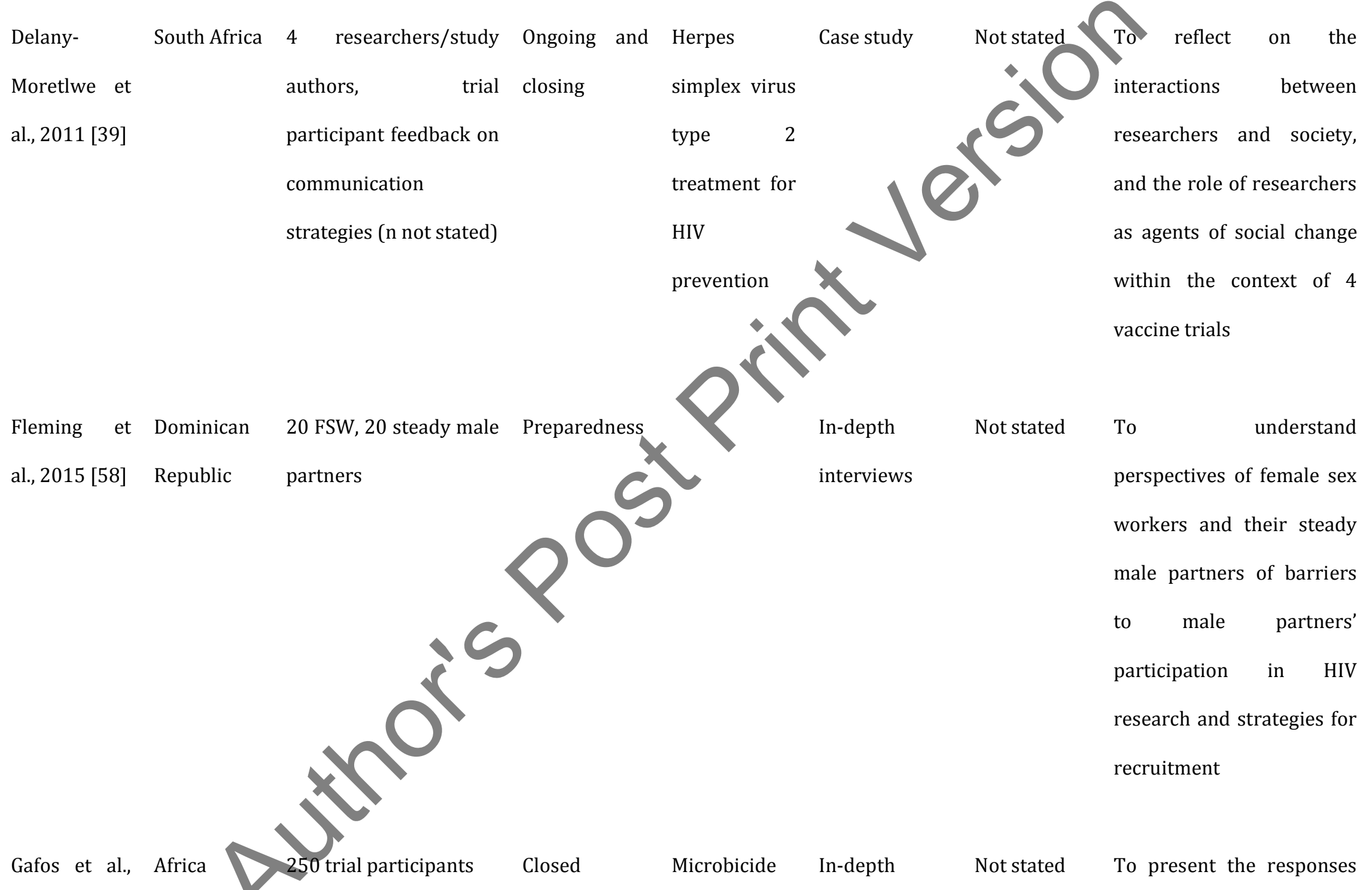




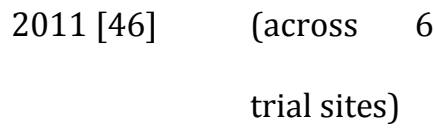

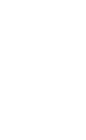
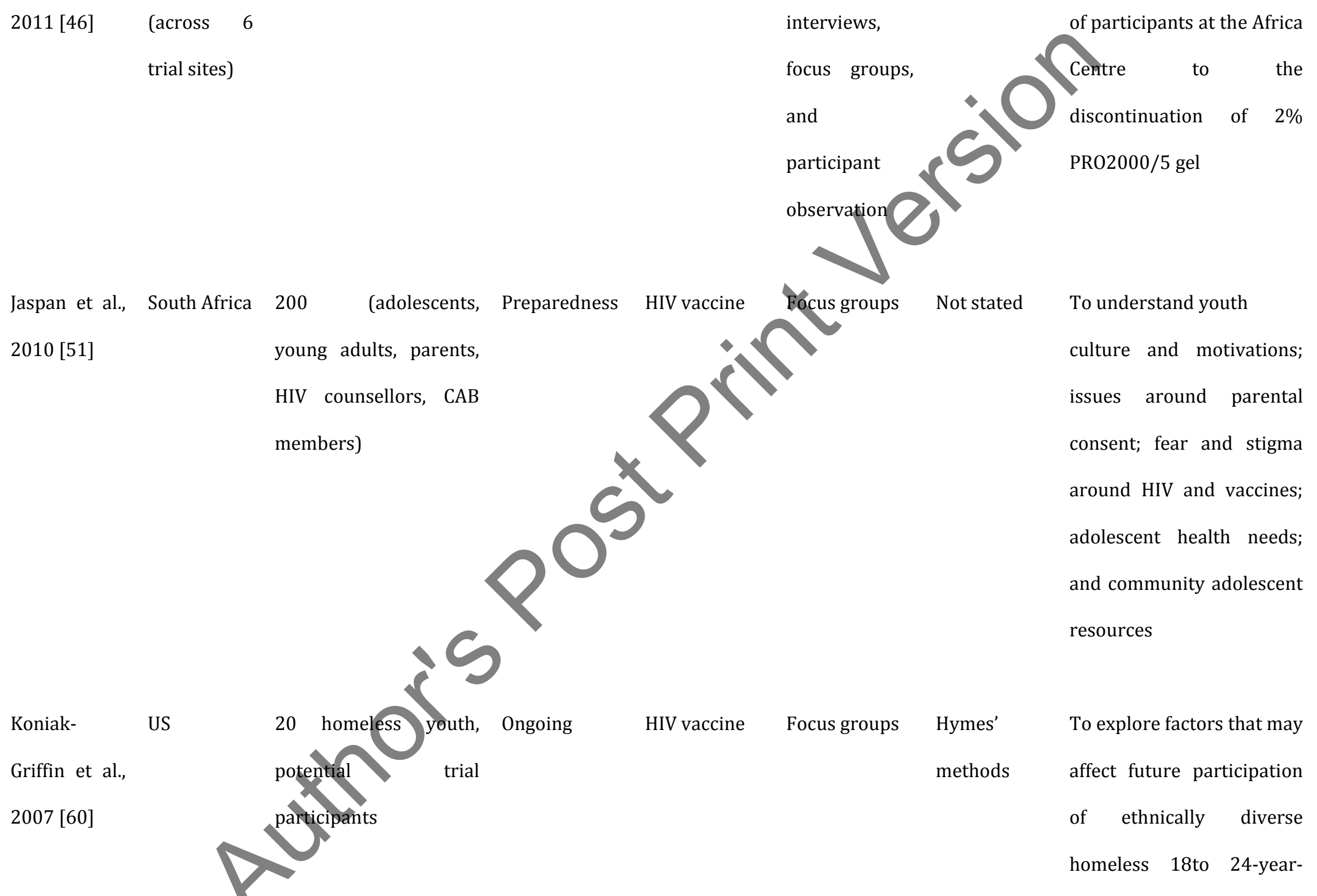


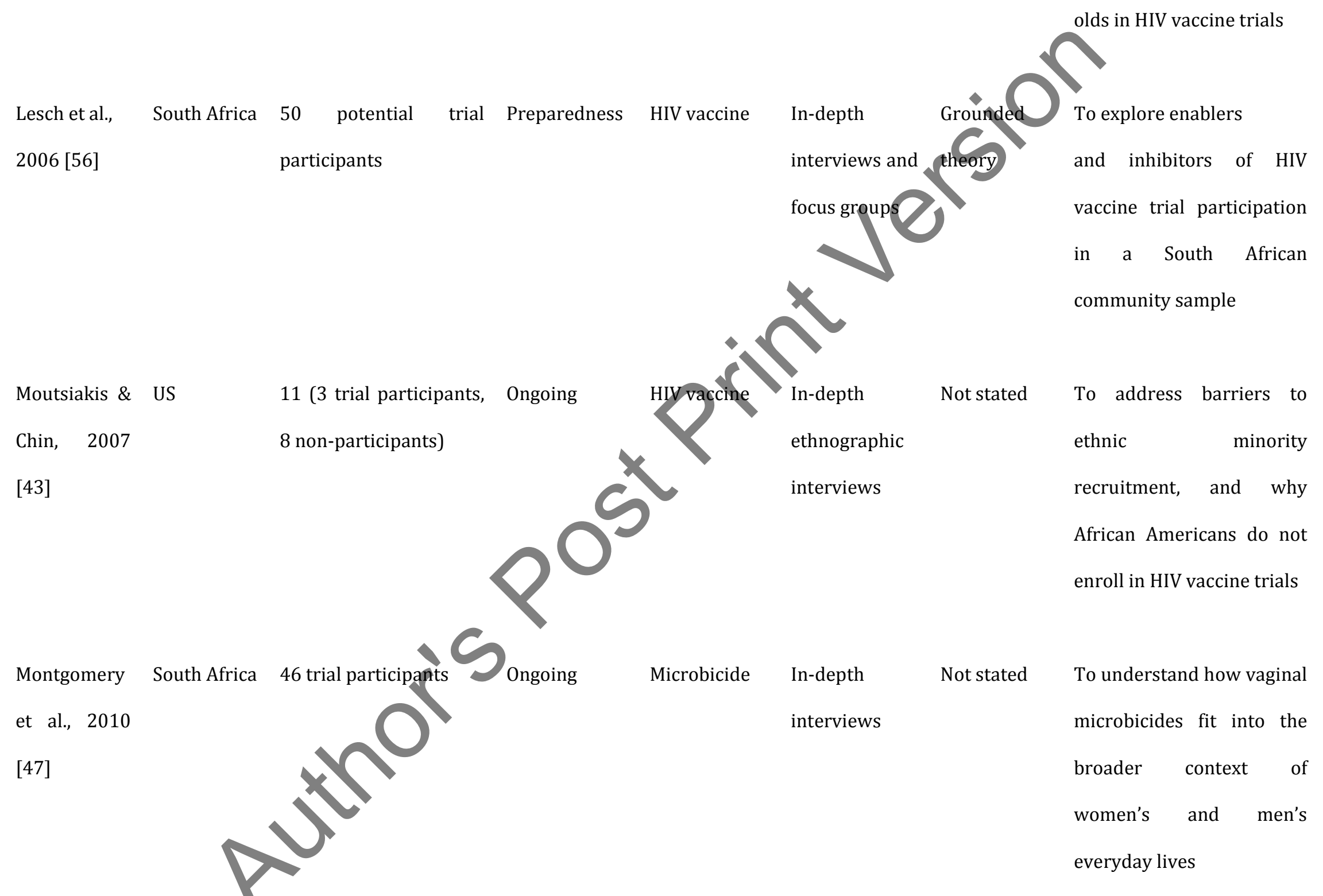




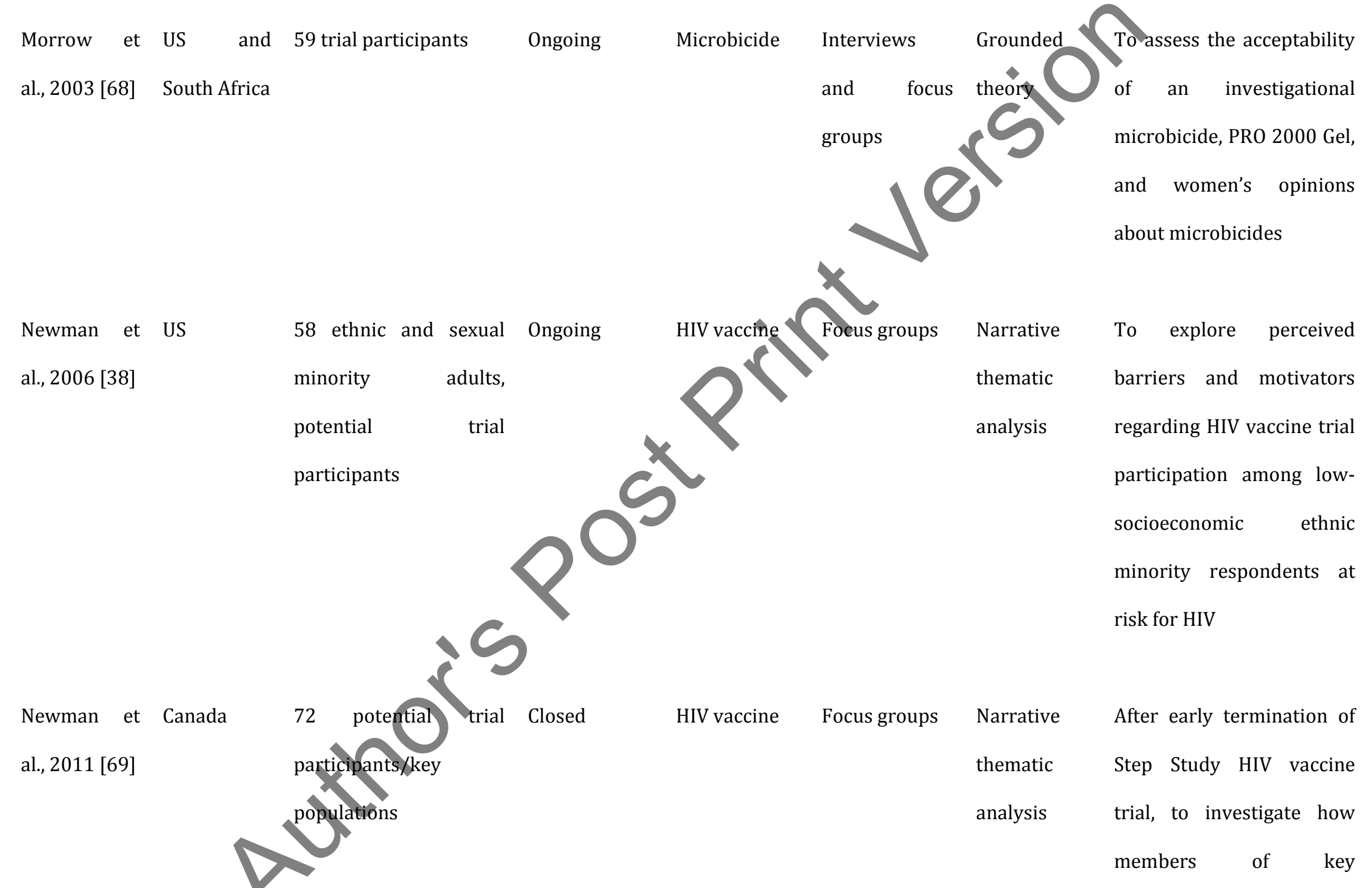




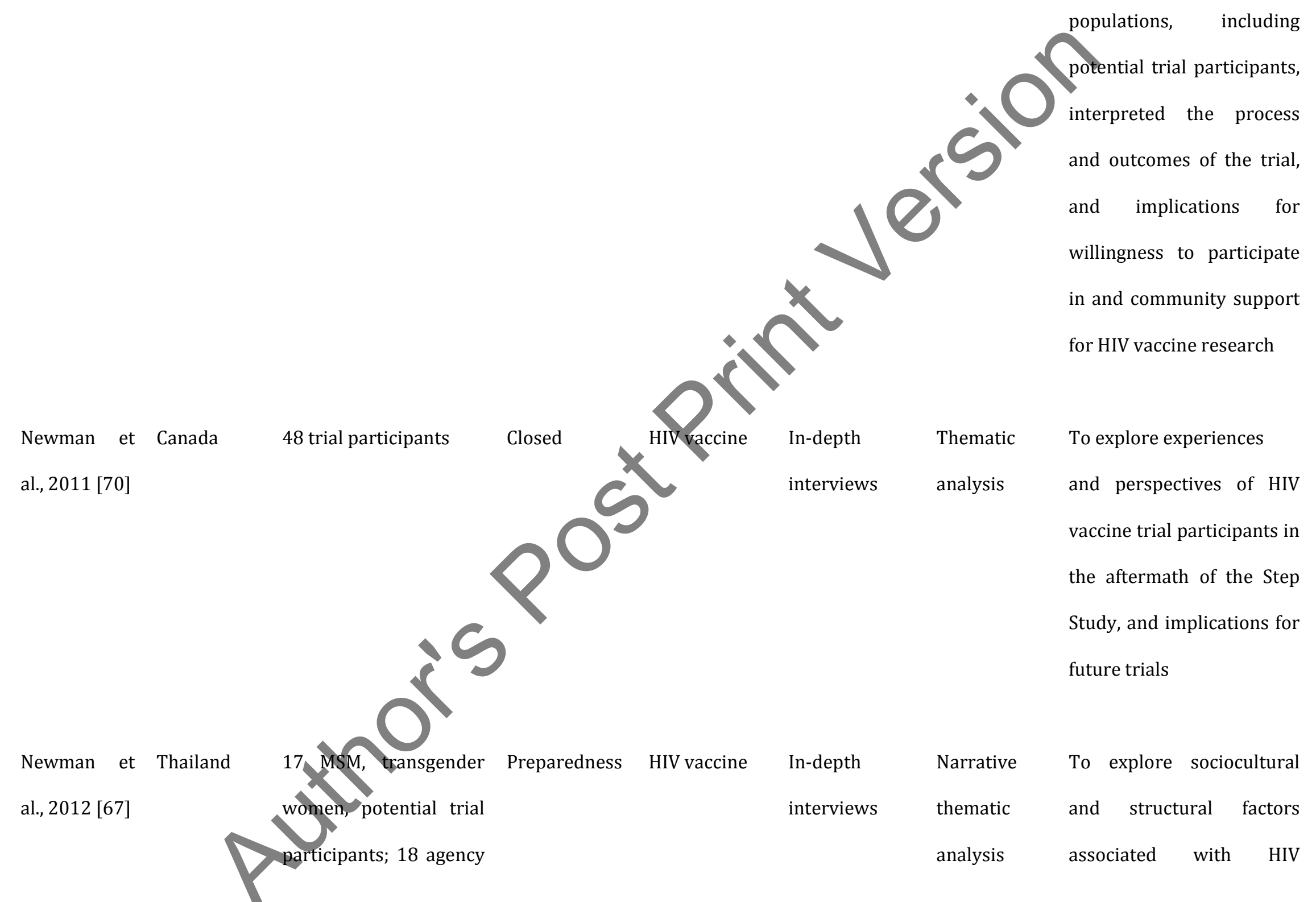


staff/advocates, $\quad 4$

health care providers

vaccine acceptability in

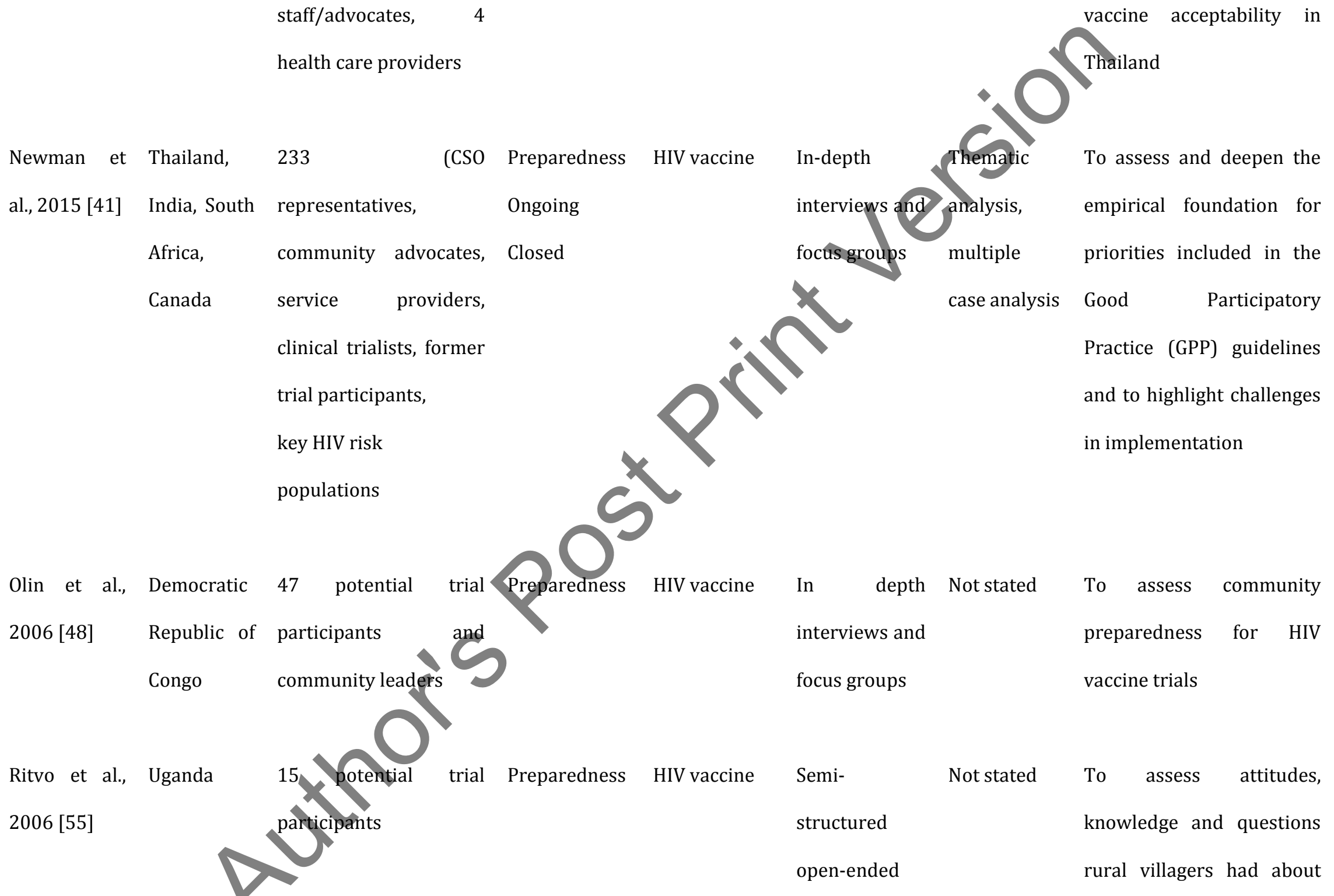




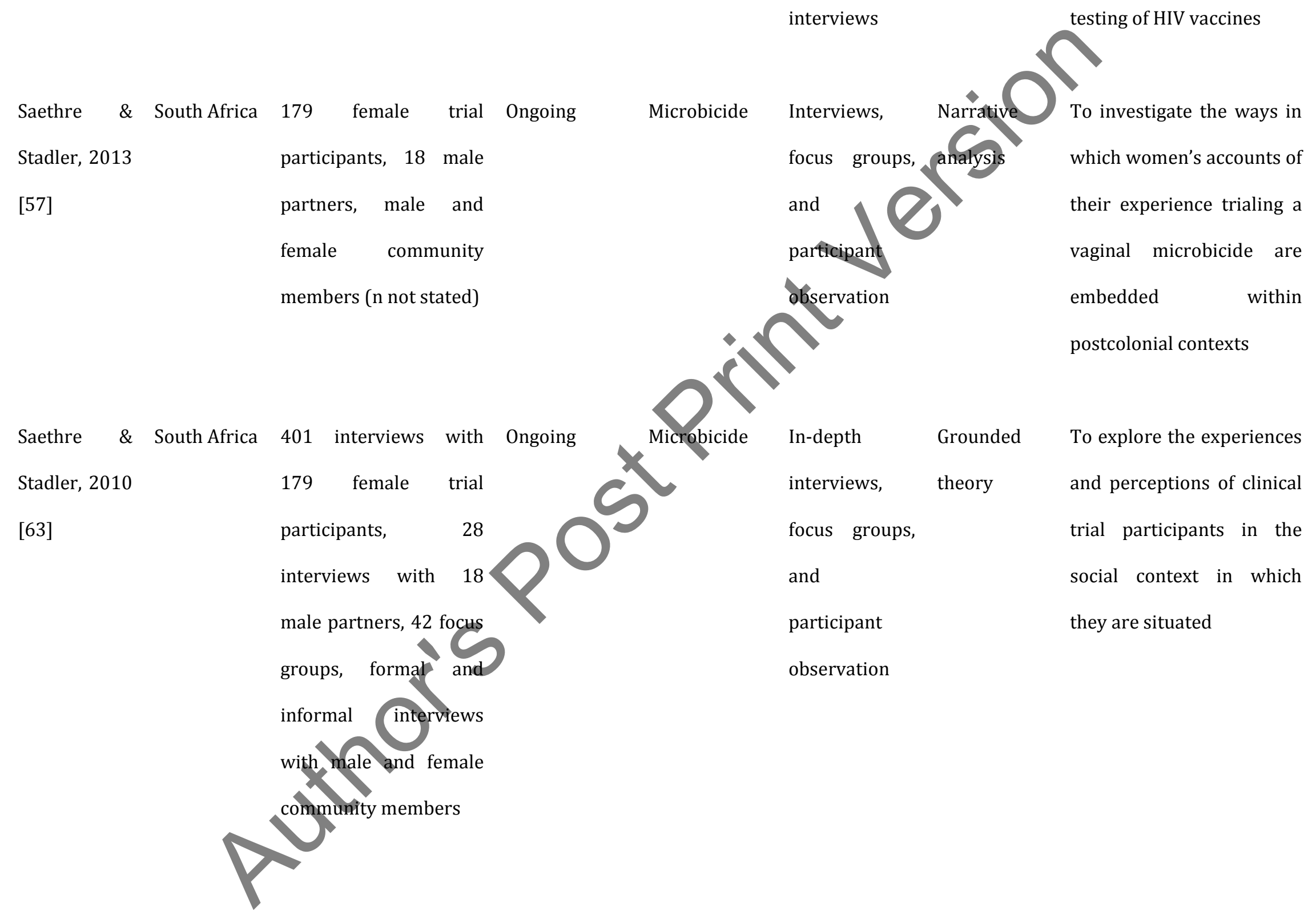




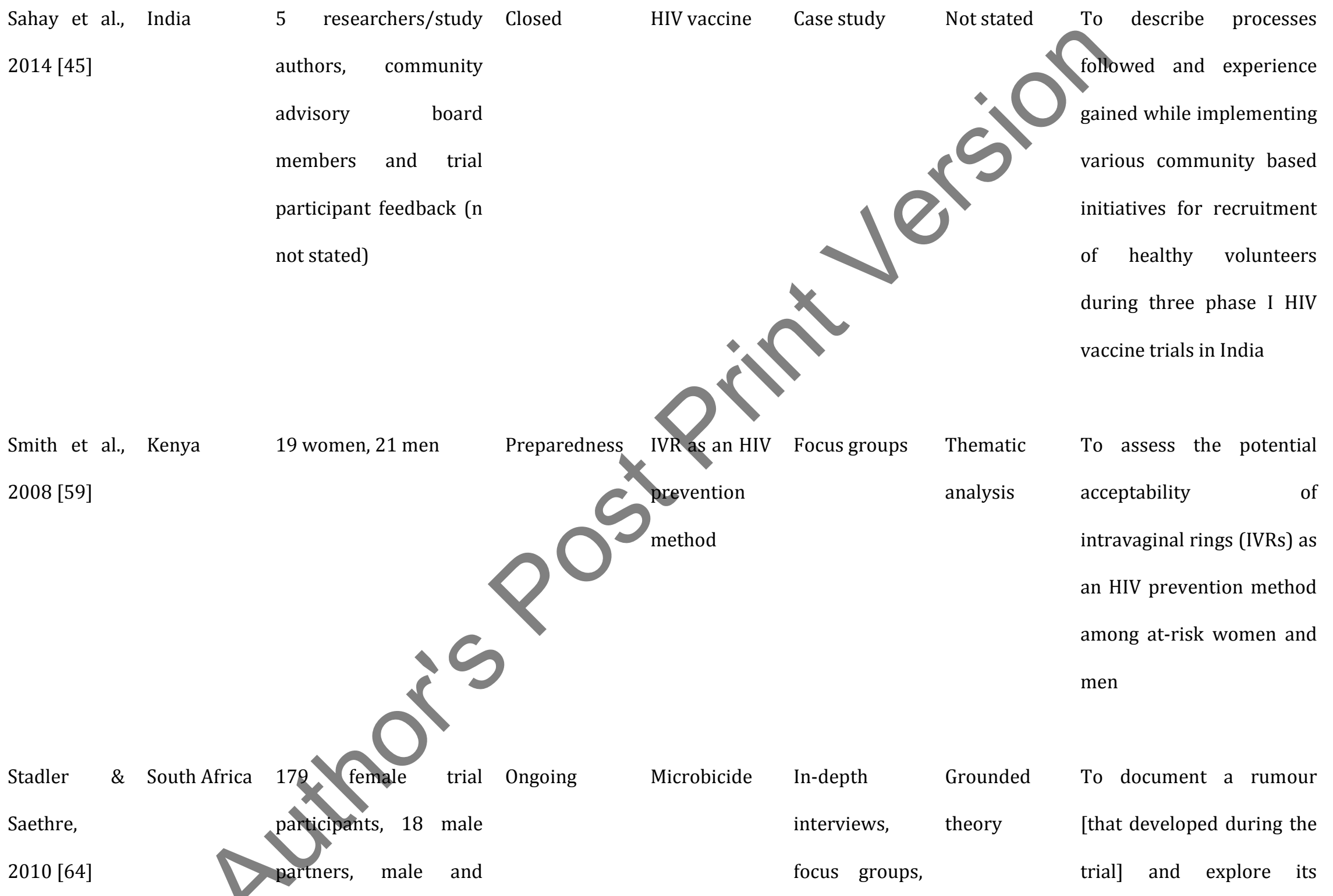


female community

members (n not stated)
(20)

Tarimo et al., Tanzania

2011 [54]

Van der Elst Kenya

et al., 2013

[37]

\section{5 trial participants}

Ongoing
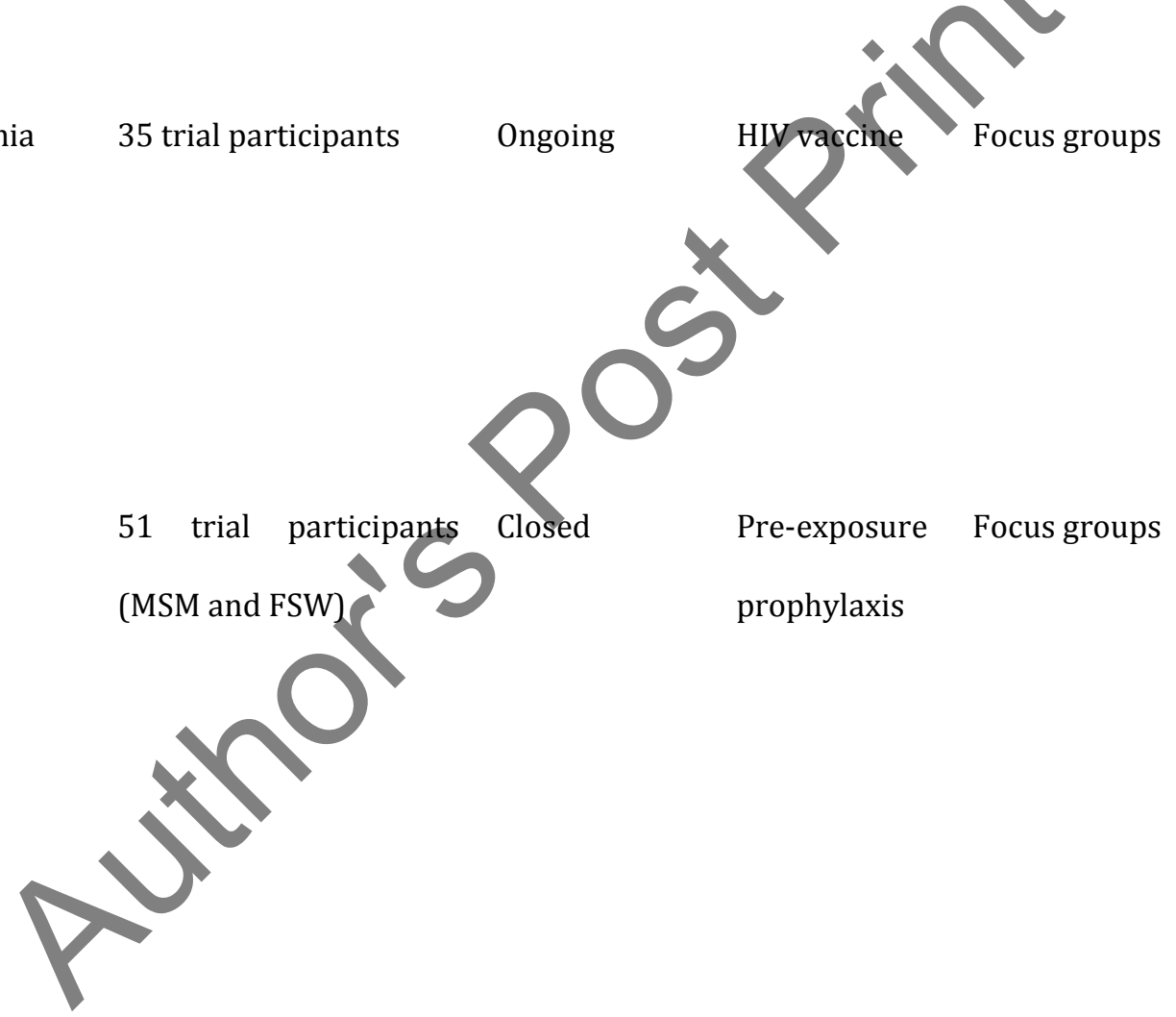

and

participant

\section{observation}

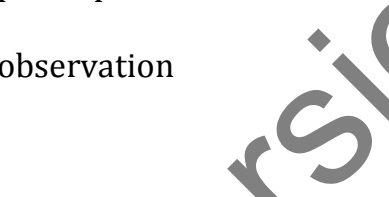

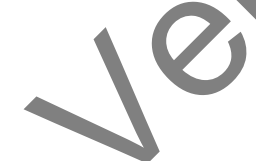

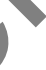

divergent interpretations

and meanings in relation to the context of the trial and the social and economic setting at two of the trial sites (Soweto and Orange Farm) in South Africa

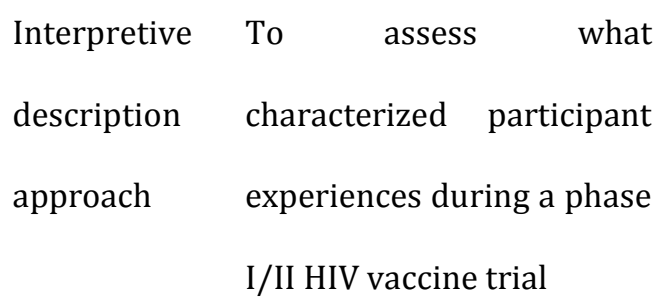

Interpretive To assess what description characterized participant approach experiences during a phase I/II HIV vaccine trial

Pre-exposure Focus groups

Framework To explore participants'

prophylaxis

\author{
perceptions of PrEP \\ acceptability, adherence \\ challenges, and develop \\ recommendations for \\ education and counseling
}




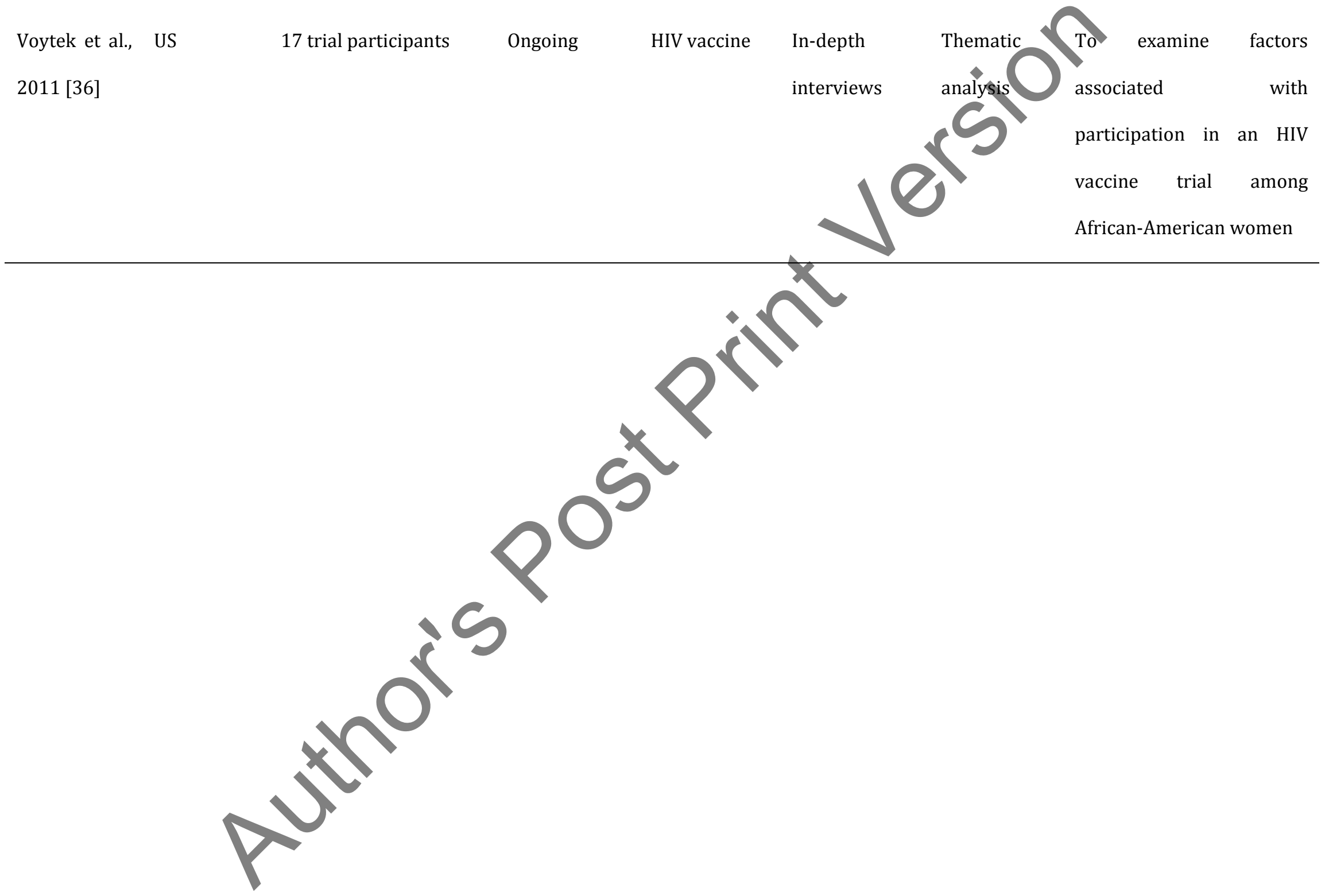


Table 2. Quality of reporting

\begin{tabular}{ll}
\hline Reporting Criteria & $\mathrm{N}(\%)(\mathrm{n}=29)$
\end{tabular}

\section{Domain 1: Research Team and Reflexivity}

Personal Characteristics

1. Interviewer or facilitator identified

$9(31)$

2. Credentials

$10(34)$

3. Occupation

4. Gender

5. Experience and training

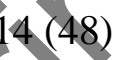

Relationship with Participants

6. Relationship established before study start

$14(48)$

7. Participant knowledge of the interviewer

$6(21)$

8. Interviewer characteristics

$1(3)$

\section{Domain 2: Study Design}

Theoretical Framework

9. Methodological orientation and theory

$22(76)$

Participant Selection

10. Sampling method

$24(83)$

11. Method of approach

$22(76)$ 
12. Sample size

13. Number or reasons for non-participation

Setting

14. Setting of data collection

15. Presence of non-participants

16. Description of sample

Data Collection

17. Interview guide

18. Repeat interviews

19. Audio/visual recording

20. Field notes

21. Duration of interviews or focus groups

22. Data saturation
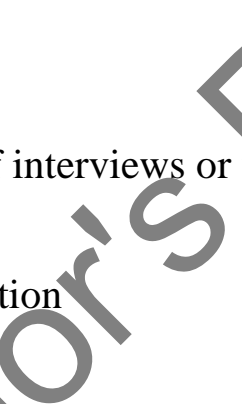

23. Transcripts returned to participants

\section{Domain 3: Analysis and Findings}

DataAnalysis

24. Number of data coders

$16(55)$

25. Description of the coding tree

$24(83)$
22 (76)

$10(34)$

$26(90)$

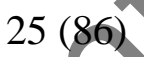

,

$13(45)$

$26(90)$

13 (46)

15 (52)

5 (17)

$2(7)$

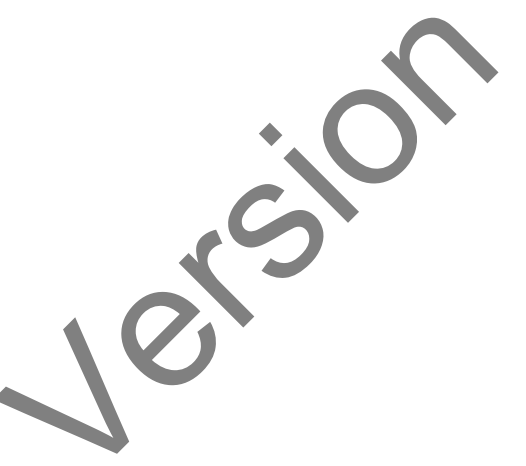


26. Derivation of themes

27. Software used

28. Participant checking

Reporting

29. Quotations presented

30. Data and findings consistent

31. Clarity of major themes

32. Clarity of minor themes
$26(90)$

20 (69)

$5(17)$

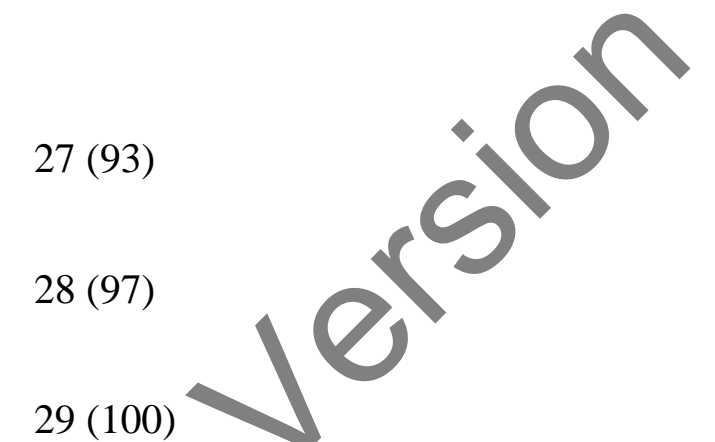

